Nouvelles perspectives en sciences sociales

Revue internationale de systémique complexe et d'études relationnelles

\title{
Gouverner la complexité? Étude du paysage comme expérience de gouvernance pour les acteurs publics locaux (Blois, Nevers, France)
}

\section{Lolita Voisin}

Volume 10, numéro 1, novembre 2014

Sur le thème du territoire

URI : https://id.erudit.org/iderudit/1028438ar

DOI : https://doi.org/10.7202/1028438ar

Aller au sommaire du numéro

Éditeur(s)

Prise de parole

ISSN

1712-8307 (imprimé)

1918-7475 (numérique)

Découvrir la revue

Citer cet article

Voisin, L. (2014). Gouverner la complexité? Étude du paysage comme expérience de gouvernance pour les acteurs publics locaux (Blois, Nevers, France). Nouvelles perspectives en sciences sociales, 10(1), 115-162. https://doi.org/10.7202/1028438ar
Résumé de l'article

Cet article propose d'interroger les processus de construction d'une action publique territorialisée. À partir du cas de trois villes moyennes françaises, l'article souligne la mobilisation d'un sujet particulier par les acteurs publics locaux : le paysage. Pourquoi et comment le paysage est-il mobilisé? En observant les pratiques et les discours des acteurs, c'est l'organisation territoriale en construction qui apparaît en filigrane, notamment à travers les communautés d'agglomération. L'article se propose d'ouvrir de nouveaux questionnements concernant la capacité du paysage à gouverner la complexité des phénomènes spatiaux contemporains et des attentes sociales et politiques démultipliées. 


\title{
Gouverner la complexité? Étude du paysage comme expérience de gouvernance pour les acteurs publics locaux (Blois, Nevers, France)
}

\author{
LoLITA VoIsIN \\ École nationale supérieure de la nature et du paysage, Blois \\ CNRS CITERES, Université François-Rabelais, Tours
}

\begin{abstract}
Ces dernières années ont vu la multiplication de dispositifs

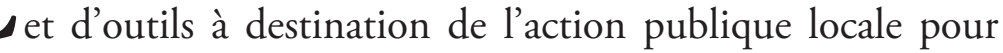
organiser le développement territorial. Ces nouvelles procédures vont de pair avec la croissance de la capacité d'action, notamment en termes de compétences, des collectivités locales, depuis les mouvements successifs de décentralisation. Si un effort croissant est porté à éclaircir ces mécanismes et à trouver la cohérence d'une action publique devenue procédurière, la pratique demeure souvent illisible et complexe pour les acteurs mêmes du territoire. Pourtant, l'analyse de l'action publique contemporaine insiste de plus en plus sur l'épaisseur nouvelle du local et sur l'entrée de nouveaux acteurs au sein de l'organisation politique locale. Or, à l'image de l'ensemble de l'action publique française, perdurent sur les territoires deux logiques en matière de développement territorial : l'une issue de l'État central, garant de l'égalité de territoires différenciés et de la cohésion nationale, l'autre venue des territoires eux-mêmes, sujets à la concurrence et à la
\end{abstract}


spécialisation, devenus légitimes quant aux choix à effectuer pour leurs propres perspectives. Or, la structure urbaine est devenue multipolaire et métropolisée, son fonctionnement n'est appréhendé qu'en partie par les acteurs, de nouveaux phénomènes spatiaux et sociaux restent encore illisibles, voire "invisibles» pour l'action publique nationale et territoriale.

Ainsi, dans ce contexte, les acteurs publics locaux, notamment les élus et les techniciens des collectivités locales, sont invités à appliquer des injonctions, venues $d u$ haut, et, en même temps, à inventer un projet local cohérent et singulier, initié puis porté par le bas. Or, à ce titre, la notion de paysage est particulièrement fertile pour observer ce processus : dans un environnement national où la question du paysage est avant tout présente dans l'arsenal réglementaire de la protection (patrimoine, écologie, etc.), davantage perçue comme une contrainte par les porteurs de projets, notamment par les acteurs publics, qu'en est-il lorsque le paysage est directement invoqué et mobilisé par une collectivité, lorsqu'il est appelé par le bas? Existe-t-il des cas où il deviendrait un levier d'expérimentation pour une pratique interne de projet territorial innovant? La mobilisation du paysage pourrait-elle alors revenir à être un outil de développement territorial, dans un processus de territorialisation, permettant d'absorber de nouvelles problématiques spatiales et de nouvelles questions d'organisation politique? Peut-on aller jusqu'à penser que, dans certains cas, reprenant le titre d'un ouvrage outreatlantique dirigé par Mario Bédard ${ }^{1}$, la mobilisation du paysage peut se révéler être un "projet politique» local?

C'est en établissant la comparaison des trajectoires singulières de trois villes moyennes françaises que ces questions ont été soulevées, argumentées par une étude de terrain approfondie sur l'une de ces agglomérations : la ville de Blois (Loir-et-Cher). Le choix de s'intéresser aux villes moyennes s'explique notamment par le fait que la mise en place du projet territorial de ce type de

Mario Bédard, "Habiter la Terre. Le paysage, un projet politique», dans Mario Bédard (dir.), Le paysage : un projet politique, p. 1-12, Québec, Presses universitaires du Québec, 2009. 
villes, isolées ${ }^{2}$, comprend nécessairement une réflexion sur le centre de l'agglomération mais également sur ses franges, aux contours plus ou moins flous. Parallèlement, la construction intercommunale restant assez inégalement avancée selon les agglomérations, l'organisation politique locale est également source de projet. Comprendre conjointement le projet territorial et le projet politique d'une ville moyenne, à travers la notion de paysage, permet ainsi de souligner des processus de territorialisation plus ou moins singuliers, et d'amender une réflexion toujours prégnante sur les politiques d'aménagement territorial. Peut-on regarder certaines formes de mobilisation du paysage comme une expérimentation pour gouverner la complexité territoriale?

\section{Les politiques de développement territorial et la saisie de la notion de paysage au sein de villes moyennes ligériennes}

La manière dont les acteurs des villes moyennes s'organisent et construisent le territoire reste difficile à appréhender directement. C'est en observant les pratiques et les discours concernant le territoire, en même temps que les signes d'évolution spatiale et sociale, que l'on peut tenter de dessiner une «carte» de l'impression territoriale des acteurs à un instant précis. C'est ce que nous présentons ici en enquêtant sur la mobilisation de la notion de paysage, dans deux agglomérations de taille moyenne, Blois et Nevers. Avant de détailler les résultats territorialisés, nous revenons sur le contexte de l'usage de la notion de paysage dans les collectivités locales.

1.1. L'action publique paysagère dans les collectivités locales: une réponse descendante aux injonctions nationales ou une forme émergente de stratégie locale?

S’interroger sur la mobilisation du paysage par les acteurs des territoires revient à s'interroger sur les multiples formes que peut prendre cette notion. En effet, elle est complexe, instable, mouvante (ou changeante) dans le temps et l'espace, c'est une notion

\footnotetext{
2 Nous entendons ici non rattachées à l'influence directe d'un pôle métropolitain.
} 
ancienne, dont les tentatives de définitions évoluent sans cesse. Le paysage est un de ces "objets hybrides ${ }^{3}$ ", une notion aux contours flous, qui suppose une interprétation toujours singulière de son sens. Ce constat est à établir en parallèle de celui de la recomposition spatiale et politique des territoires. En effet, les collectivités locales, notamment les communes et les intercommunalités, sont amenées à tester de nouveaux modes de gestion des territoires, plus performants, plus économes, mais également plus démocratiques, en tout cas plus représentatifs des électeurs et des usagers des territoires. Ainsi, qu'en est-il quand les collectivités locales mobilisent le paysage : est-ce un mouvement de traduction descendant ou la mise en ouvre d'une stratégie plus locale? Afin de répondre à cette question, il convient de revenir sur une lecture diachronique de l'institutionnalisation de la notion de paysage et de la réglementation qui l'accompagne, ceci après avoir évoqué la polysémie du terme. Nous pourrons ainsi conclure sur l'application certes descendante du paysage en France, mais également sur l'importance de la part ascendante, c'est-à-dire venue directement des acteurs publics locaux.

\subsection{A. Un enjeu politique du paysage}

Réaliser une synthèse de la "problématique du paysage» à travers les grands courants qui définissent une théorie du paysage complexe et polysémique n'est pas chose aisée. Jean-Marc Besse s’y est essayét. Il dénombre cinq courants dont il reconnaît la légitimité propre et les relations. Ces courants explorent chacun les ramifications et les sentiers empruntés par une pensée du paysage sans cesse investie par de nouveaux acteurs; ils fournissent une cartographie complexe et croisée, non exhaustive, des pratiques et des valeurs sous-tendues par la notion de paysage. L'acceptation, même provisoire, de ces juxtapositions et superpositions est le préalable nécessaire aux travaux qui viennent s'y ajouter. Ainsi, ces différentes approches sont 1) celle de la

3 Bruno Latour, Nous n'avons jamais été modernes. Essai d'anthropologie symétrique, Paris, La Découverte, 1991.

4 Jean-Marc Besse, Le goût du monde. Exercices de paysage, Arles, Actes Sud/ ENSP, coll. «Paysage», 2009. 
représentation culturelle (portée par des historiens et des philosophes de l'art, comme Alain Roger ou encore Georg Simmel, Anne Cauquelin); 2) celle inspirée de la géographie culturelle, qui voit dans le paysage une ouvre collective des sociétés (l'approche d'historiens et de théoriciens, par exemple de l'américain John Brinckerhohh Jackson, mais aussi Éric Dardel, Jean Brunhes, Pierre Deffontaines ou encore Augustin Berque); 3) celle qui voit dans le paysage un espace d'expériences sensibles rebelles aux diverses formes possibles d'objectivation (par exemple les anthropologues et les historiens des sensibilités : Pierre Sansot, Alain Corbin, ainsi que les philosophes de l'esthétique : Ernst Cassirer, Joachim Ritter...); 4) celle inspirée par les sciences écologiques et la connaissance du vivant, qui voit le paysage comme un complexe systémique (par exemple les écologues, comme Georges Bertrand et certains géographes); 5) enfin celle propre aux paysagistes et aux professionnels de l'aménagement, qui voient dans le paysage la matière du dessin, de la transformation de l'espace, la matière à projeter, le socle préalable à toute forme de programme d'action (citons par exemple des paysagistes maîtres d'œuvre ou des urbanistes : Gilles Clément, Michel Corajoud, Michel Desvigne, etc., mais aussi des philosophes comme Sébastien Marot).

La dimension politique du paysage se situe à la croisée de ces différentes approches. En effet, chaque fois que le paysage est appréhendé, que ce soit dans un discours d'acteurs, par exemple, ou dans le cadre d'une politique publique locale, chacune de ces tendances du paysage peut être comprise, éventuellement se croiser avec d'autres, l'une dominant l'autre, l'autre complétant l'une. On ne peut regarder la notion de paysage mobilisée par un acteur comme une valeur unique et universelle. Anne Sgard avance que :

Le paysage se situe à une "échelle humaine», celle des pratiques quotidiennes, de l'horizon ordinaire, et de la co-présence souvent conflictuelle. Comment est-ce que l'individu peut dès lors arbitrer entre les valeurs marchandes, mesurables, et les valeurs symboliques? Entre des valeurs individuelles et le souci du bien commun? Ce sont les 
obstacles sur lesquels buttent au quotidien les politiques publiques qui cherchent à mettre le paysage au centre de leur action ${ }^{5}$.

\subsection{B. Lecture diachronique de l'action publique paysagère dans les collectivités locales au regard de l'institutionnalisation $d u$ paysage}

Or, on assiste aujourd'hui à une multiplication croissante de la mobilisation de la notion de paysage dans l'action publique : soit au titre de l'application de politiques nationales (classement au titre de la loi du 2 mai 1930, ou volet paysager des permis de construire par exemple), voire internationales (patrimoine mondial de l'UNESCO par exemple); mais également dans les discours des acteurs locaux, dans lesquels le paysage devient un argument de promotion du territoire et de ses atouts.

Or, le paysage n'est pas neutre : on ne «suspend pas le sens [du paysage]" rappelle Augustin Berque, à propos des réglementations patrimoniales qui "gèlent» celui-ci ${ }^{6}$. Quand le paysage est saisi par un acteur, c'est par le biais de sa propre représentation, de son histoire, de sa formation, mais également de ses intentions à un instant précis, conscientes ou souterraines. L'argument du paysage peut porter différents objectifs pour les acteurs d'un territoire : il peut être appelé pour définir «une identité territoriale», notamment par la définition d'unités paysagères ${ }^{7}$. À l'échelle d'une nation, le paysage a souvent servi de références patriotiques : les travaux de François Walter ont montré comment

5 Anne Sgard, "Une "éthique du paysage” est-elle souhaitable?", Vertigo, vol. 10, $\mathrm{n}^{\circ}$ 1, Dossier Éthique et Environnement à l'aube du XXI' siècle: la crise écologique implique-t-elle une nouvelle éthique environnementale?, 2010, [en ligne] http://vertigo.revues.org/9472, site consulté en mars_2011.

6 Augustin Berque, "Douter du paysage», dans Jean-Pierre Le Dantec (dir.), Jardins et Paysage. Textes critiques de l'Antiquité à nos jours, Paris, Larousse, 1996.

7 Cette appellation provient de la méthode des atlas de paysage, outil de caractérisation des paysages, en voie de généralisation sur l'ensemble du territoire national (Bertrand Folléa, Guide des plans de paysage, des chartes et des contrats, Ministère de l'Aménagement du territoire et de l'environnement, 2001). 
les nations en construction au $\mathrm{XIX}^{\mathrm{e}}$ siècle usaient de figures paysagères pour s'affirmer, se légitimer et être reconnues ${ }^{8}$.

Il peut être vécu comme une ressource spécifique ou encore un "panier de biens ${ }^{9}$ » mais il peut aussi

alimenter des discours tendant au refus de tout changement, qu'il s'agisse de l'appel à un paysage patrimonialisé, figé, ou au bien connu syndrome $\mathrm{Nimby}^{10}$. [Il peut même] justifier des logiques de ségrégation socio-spatiale [...], des logiques de repli grâce au blocage foncier, [...] des logiques de fermeture ${ }^{11}$.

Il est en tout cas fréquent que le paysage soit un argument d'opposition, par exemple dans le cas d'une résistance à un projet éolien ${ }^{12}$.

L'arsenal réglementaire français, mis en place en faveur du paysage, depuis les lois du 21 avril $1906^{13}$ et du 2 mai $1930^{14}$ sur la protection du patrimoine et des sites, jusqu’à la récente ratification de la Convention européenne du paysage par la France en 2006, constitue un cadre d'action plus ou moins restrictif, avec lequel les acteurs locaux négocient pour une action territorialisée. Les lois, décrets et instruments concernant directement le paysage se sont élargis de la notion de patrimoine monumental puis naturel à la notion de paysage ordinaire, quotidien, telle que revendiquée dans la Convention européenne du paysage.

$8 \quad$ François Walter, Les figures paysagères de la nation. Territoire et paysage en Europe (XVI $-X X^{e}$ siècles), Paris, Éditions de l'EHESS, 2004.

9 Bernard Pecqueur, "Qualité et développement territorial : l'hypothèse du panier de biens et de services territorialisés", Économie rurale, n 261, 2001, p. 37-49.

10 Not In My Back Yard [pas dans mon arrière-cour, dans mon jardin], mouvement de résistance ou d'opposition à un changement pour lequel une population doit sacrifier un partie de ses avantages ou de ses biens.

11 Anne Sgard, "Une "éthique du paysage" est-elle souhaitable?", op. cit.

12 Marie-Josée Fortin, "Le paysage, cadre d'évaluation d'une société réflexive», dans Martine Berlan-Darque, Yves Luginbühl, Daniel Terrasson (dir.), De la connaissance des paysages à l'action paysagère, Versailles, Éditions Quae, p. 223231, 2007.

13 Loi du 21 avril 1906 organisant la protection des sites et monuments naturels de caractère artistique.

14 Loi du 2 mai 1930 ayant pour objet de réorganiser la protection des monuments naturels et des sites de caractère artistique, historique, scientifique, légendaire ou pittoresque. 
Cependant, la réglementation française sur le paysage reste davantage construite autour de sa dimension conservatrice, élitiste et patrimoniale. Cette tendance évolue dans les années 1970, après la loi d'orientation urbaine et foncière (1967) et la création du Centre National d'Études et de Recherche sur le Paysage (1972) qui a pour mission de mettre en place des méthodes d'approche de la question paysagère à destination des administrations. Ces deux événements fondent ainsi «le grand paysage», aussi appelé alors "paysage d'aménagement ", avec une dimension planificatrice, le paysage devant mettre en valeur les espaces vides, les espaces non bâtis, les «réserves » futures de la ville. Ainsi, cette nouvelle dimension de paysage planificateur est directement appliquée aux villes moyennes, quand leurs schémas directeurs d'aménagement et d'urbanisme (SDAU) dessinent les grands axes de l'urbanisation future, prenant parfois appui sur la «structure verte» du territoire pour en dessiner l'avenir. C'est le cas à Blois par exemple, lorsque la Chambre de Commerce et d'Industrie signe en 1971 un plan d'extension (voir la figure 1), où le paysage fluvial et le paysage du plateau de la Beauce organisent la structure future de la ville, notamment les entrées de ville. 
Figure 1

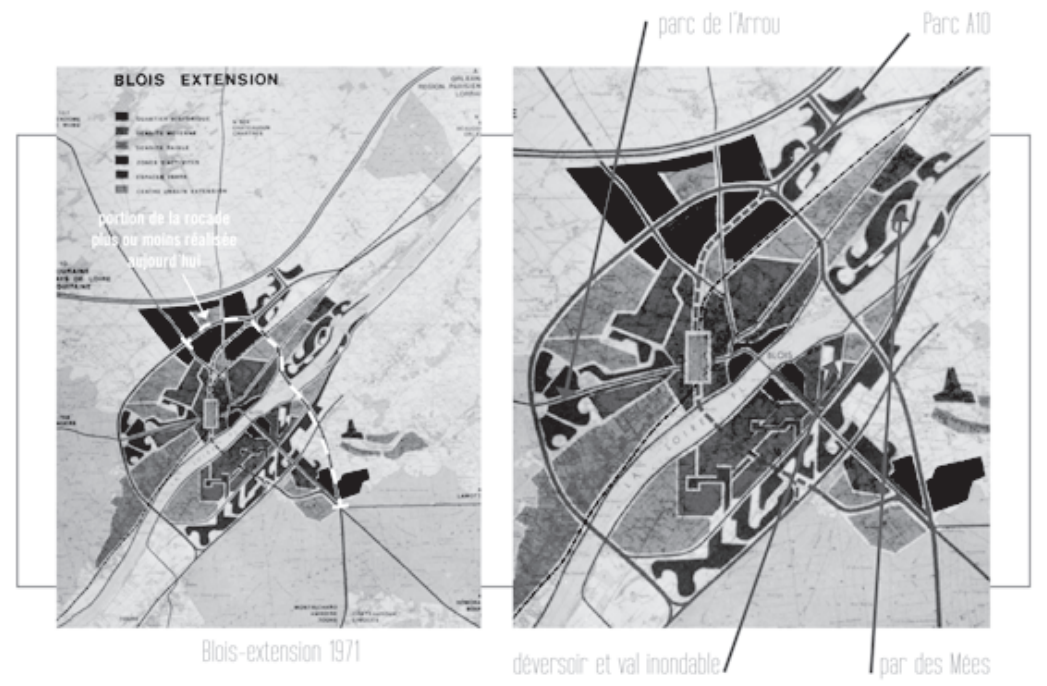

Le plan d'extension de Blois en 1971, qui préfigure le Schéma Directeur d'Aménagement et d'Urbanisme de 1974. Le paysage est le support d'organisation de la ville future, notamment la vallée et les entrées de ville (formes géométriques sur le plan). Source : Blois, Ville moyenne

Il n'y a pas eu alors de véritable institutionnalisation du paysage. Le paysage a été balloté d'un ministère à l'autre, entre l'équipement et la culture, l'environnement et le cadre de vie, parfois même séparé en deux ${ }^{15}$. Par la suite, de nombreux essais ont été engagés pour une "politique de paysage d'État», transversale et intégrée, à l'image des missions interministérielles de la DATAR ${ }^{16}$. Aujourd'hui, les politiques publiques de paysage semblent revenues à une dimension moins ambitieuse, voire

$\overline{15}$ La création du ministère de l'environnement (1971) a provoqué une certaine réticence de la part du ministère de la culture. Le premier ministre a alors arbitré et séparé deux types de paysages : le paysage naturel à l'environnement, le paysage urbain à la culture, tendance toujours perceptible aujourd'hui dans les administrations déconcentrées.

16 Alexis Pernet, Le grand paysage en projet entre trajectoires institutionnelles et territoires vécus - L'expérience de l'atelier des paysages en vallée de l'Ance (Parc naturel régional Livradois-Forez, Auvergne, France), thèse de doctorat en géographie, Université Panthéon Sorbonne-Paris 1, 2011. 
deviennent incertaines ${ }^{17}$. Ainsi, si l'on peut dire que l'État a vu un jour le paysage comme projet politique dans les années 1980, cette période volontariste semble maintenant achevée. C'est le constat que fait Bernard Barraqué lors de la réédition en 2005 de son travail de recherche "Paysage et administration", très éclairant sur la question, lorsqu'il s'interroge quant à la trajectoire de la notion de paysage au sein des doctrines de l'aménagement de la France. L'auteur reconnaît que, peu après la date de la première écriture de son étude (1985), relatant de nombreux efforts politiques, par des personnalités comme Pierre Sudreau ${ }^{18}$ ou Robert Poujade, pour une politique de paysage «plus incitative et intégratrice», on semblait être revenu "dans une période d'incertitudes inter-administratives, professionnelles voire scientifiques». Il se demandait également "si les paysagistes n'étaient pas tentés de renoncer à l'idée d'exercer leur métier dans la fonction publique et la planification, l'aménagement, préférant encore et toujours la création en libéral ${ }^{19}$, ce qui démontrerait une difficulté pour le paysage à s'incarner politiquement dans les institutions centrales et décentralisées.

Ainsi, la trajectoire du paysage dans l'administration de l'État montre à quel point celui-ci a subi de nombreux allers et retours, a changé de nombreuses fois de ministères, a fait l'objet de missions transversales pilotées par une administration gestionnaire, pour revenir maintenant à une conception principalement réglementaire. Ces nombreux croisements sont les témoins de l'incertitude, pour les acteurs publics, que recouvre cette notion et de la difficulté pour les acteurs de terrain d'incarner une politique restée centralisée. À titre d'exemple, la loi Paysage de 1993 prévoit la création de "directives de protection et de mise en valeur des paysages", mais celles-ci sont élaborées à l'initiative

17 Bernard Barraqué, Paysage et administration, Mission de la recherche urbaine, MEDD-Direction de la Nature et des Paysages, 2005 [1985].

18 D'ailleurs maire de Blois (1971-1989) et député de Loir-et-Cher (1967-1981).

19 Bernard Barraqué, op. cit., p. 133. 
de l'État et approuvées par lui, les collectivités étant seulement consultées dans ce processus ${ }^{20}$.

Depuis la loi Paysage de 1993, le ministère en charge du paysage a davantage un rôle de conseiller et de centre de ressources que de législateur. Il organise la publication de documents destinés à transmettre les orientations politiques en matière de paysage et à fournir un cadre méthodologique relatifs aux outils prévus par la loi (atlas de paysage, plans de paysage), face à la diversité des interprétations possibles. Le Bureau des Paysages coordonne également des missions thématiques sur certains sujets récurrents pour lesquels le paysage est convoqué, comme l'implantation de projets éoliens ou la mise en place de jardins partagés.

Localement, ce sont les services déconcentrés de l'État (DDT ou DREAL ${ }^{21}$ ) qui contrôlent l'application d'une réglementation en faveur des paysages. Les DDT sont par exemple en charge de valider les permis de construire et sont garantes du respect de «l'intégration paysagère» des nouvelles constructions. Les Commissions départementales des sites, perspectives et paysages, présidées par le préfet de département, agissent comme des gardiens des paysages en cas de projets jugés néfastes par exemple. LÉtat confie aux agents de ses services déconcentrés le soin et le devoir d'appliquer les directives nationales, peu singularisées. Les agents déconcentrés doivent donc produire un discours et un argumentaire sur le paysage qui coïncide avec les traditions procédurales de ces administrations. Comme le confiait un agent de la Direction Départementale des Territoires du Loir-et-Cher :

Quand on [refuse un permis de construire par exemple, au nom du paysage], il faut motiver, expliquer et prouver, c'est un peu ambigu. Il faut un argumentaire, par exemple à travers le patrimoine, l'avis de

$\overline{20}$ Nathalie Blanc et Sylvie Glatron, «Du paysage urbain dans les politiques nationales d'urbanisme et d'environnement ", L'Espace géographique, vol. 2005, $n^{\circ} 1$, tome 34,2005 , p. 65-80.

21 DDT : Direction Départementale des Territoires; DREAL : Direction Régionale de l'Environnement, de l'Aménagement et du Logement. 
l'ABF ${ }^{22}$, etc. Ce n'est pas comme la sécurité publique! C'est un peu compliqué $^{23} \ldots$

Les collectivités, en devoir de faire appliquer les injonctions réglementaires à l'échelle de leur territoire, sont soumises aux services de l'État, notamment concernant les documents d'urbanisme; dans ce cadre, elles sont dans une situation de traduction d'un instrument pensé par le haut, en l'adaptant à la situation locale. Ce mouvement explique pour beaucoup les raisons des acteurs publics locaux à mobiliser la notion de paysage, mais avec de nombreux flottements et des réinterprétations. Ainsi, Catherine Caille-Cattin a montré comment les interprétations des atlas de paysages par les acteurs locaux participait à la diffusion d'une culture paysagère et au partage d'outils communs ${ }^{24}$. Cependant, Hervé Davodeau met en exergue les ambigüités des termes employés par les auteurs d'atlas de paysages, souvent paysagistes, et de la légitimité à parler d'identité paysagère quand elle ne correspond pas à une vérité vécue par les acteurs locaux ${ }^{25}$. Didier Labat a montré comment les objectifs paysagers inscrits au schéma directeur de l'aire métropolitaine de Bordeaux en 2001 sont réinterprétés, oubliés ou remis en cause selon les opportunités de la planification urbaine ${ }^{26}$.

\footnotetext{
22 Architecte des Bâtiments de France, sous l'autorité du Préfet de département, veille notamment à la bonne insertion des constructions neuves et des transformations aux abords des monuments protégés. Extrait d'un entretien avec un agent de la DDT 41, chef du service Aménagement, Connaissance et Analyse des territoires, le 8 novembre 2011.

24 Catherine Caille-Cattin, Le paysage, de l'information à l'élaboration d'une culture paysagère. Vers des médiations paysagères au service des acteurs de l'aménagement des territoires, Thèse de doctorat en géographie Université de Franche-Comté, 2005.

Hervé Davodeau, «Les atlas de paysages français ou les difficultés de concilier l'approche sensible et l'approche scientifique», Rivista Geografica Italiana, vol. 2009, n 2, 2009, p. 173-194.

26 Didier Labat, «La mise en œuvre des politiques paysagères : quand la décision publique est confrontée aux échelles de définition. L'analyse des Proximités comme outil de compréhension de la coordination de l'action paysagère", Projets de paysage, 2011, [en ligne] http://www.projetsdepaysage.fr/fr/_la_ mise_en_oeuvre_des_politiques_paysageres_quand_la_decision_publique_ est_confrontee_aux_echelles_de_definition, site consulté en janvier 2012.
} 
Ainsi, il reste difficile pour l'État d'imposer un cadre normatif applicable au paysage. Or, qui a légitimité à décider ce que doit être le paysage? Il ne faut pas minimiser la portée conflictuelle du paysage mis en politique. On peut imaginer dans cette institutionnalisation du paysage les multiples cas de conflits potentiels : imposition d'une représentation du paysage par le haut; différences de représentation selon les acteurs; utilisation du paysage comme argument de défense contre un choix d'aménagement et, ainsi, blocage au nom du paysage ${ }^{27}$; argument de défense d'un entre-soi, de gentrification ${ }^{28}$ ou de clubbisation ${ }^{29}$.

Dans ce contexte, comment les acteurs des territoires réagissent-ils à la mobilisation de la notion de paysage? Face à de nouvelles formes spatiales émiettées et à la perte des repères fonctionnels, particulièrement prégnante dans les villes moyennes, que nous apprend cette mobilisation, quand elle est locale?

\subsection{Présentation de la méthode et des territoires d'étude}

Ces réflexions émanent d'un travail de recherche mené de 2009 à 2013 sur plusieurs villes moyennes françaises ${ }^{30}$. Ces dernières subissent particulièrement de nouvelles tensions, tant spatiales que politiques. Le mitage périurbain et les nouvelles formes d'urbanisation, lâches ou étalées, préoccupent édiles, experts et citoyens. Si la régulation politique s'est depuis longtemps intéressée aux espaces ruraux et à certains aspects de l'espace urbain, elle n'avait «encore jamais abordé spécifiquement cette frange au poids croissant qu'est le tiers-espace" jusque dans les années

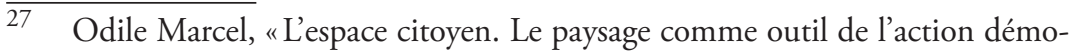
cratique», dans Mario Bédard (dir.), Le paysage: un projet politique, p. 225251, Québec, Presses de l'Université de Québec, 2009.

28 Éric Charmes, "Les politiques paysagères comme supports éthiques de la gentrification", Actes du colloque De la connaissance des paysages à l'action paysagère, 2 au 4 décembre 2004, Bordeaux, Cemagref, 2004.

29 Éric Charmes, La ville émiettée, essai sur la clubbisation de la vie urbaine, coll. «La ville en débat», Paris, Presses universitaires de France, 2011.

30 Lolita Voisin, La mobilisation du paysage par les acteurs publics locaux, un enjeu stratégique de territorialisation? Réflexions en Loire Moyenne : Blois, Nevers, Saumur, Thèse de doctorat en aménagement de l'espace-urbanisme, Tours, Université François-Rabelais, 2013. 
$2000^{31}$. Ces franges de contact incertaines sont pourtant devenues le quotidien des agglomérations, que les décideurs tentent aujourd'hui de s'approprier afin d'être en mesure d'y conduire une action et donc d'y exercer un pouvoir encore indéfini. Or, les villes moyennes ou intermédiaires ${ }^{32}$ sont souvent obligées à l'innovation car davantage délaissées des politiques publiques ${ }^{33}$. On peut analyser les territoires dans leur processus de construction, leur territorialitée ${ }^{34}$, plutôt que dans leur «état" physique, spatial. Trois villes moyennes ont fait l'objet d'une comparaison de leurs trajectoires territoriales : Blois, Nevers et Saumur (voir la figure 2).

31 Martin Vanier, "Qu'est-ce que le tiers espace? Territorialités complexes et construction politique», Revue de Géographie Alpine, tome 88, n 1, 2000, p. $105-113$.

32 Fabien Nadou, Intermédiation territoriale et spatialisation des activités économiques. Cohérences et contradictions de l'action publique locale. Investigation par la planification stratégique, thèse de doctorat, Tours, Université FrançoisRabelais, 2013.

33 Daniel Behar, Martin Vanier et Philippe Estèbe, «Pôles métropolitains : du "faire territoire" au "faire politique", ou la nouvelle bataille de l'interterritorialité», Métropolitiques, 18 mai, 2011), [en ligne] http://www.metropolitiques.eu/Poles-metropolitains-du-faire.html, site consulté en septembre 2012.

Robert Lajarge, «Pas de territorialisation sans action (et vice-versa)», dans Martin Vanier (dir.), Territoire, territorialité, territorialisation... et après?, Rennes, Presses universitaires de Rennes, 2009, p. 193-204. 
Figure 2

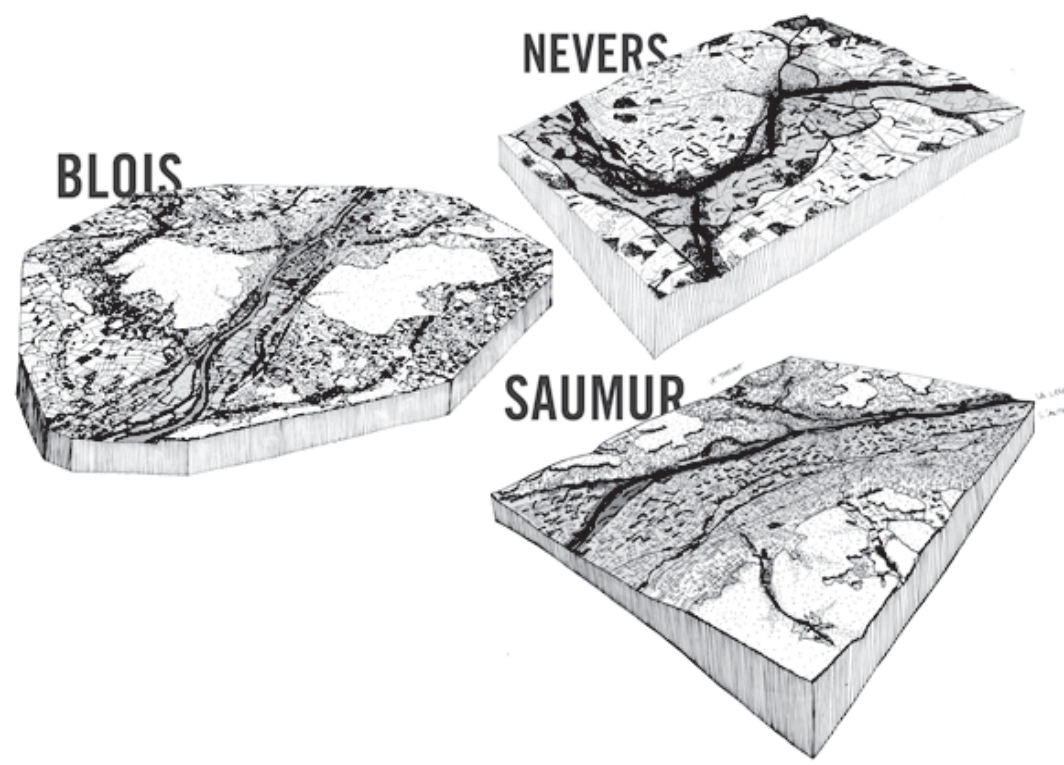

Blocs-diagrammes des trois agglomérations ligériennes ayant fait l'objet d'une analyse des trajectoires de territorialisation. Source : Lolita Voisin (La mobilisation du paysage par les acteurs publics locaux...)

Pour regarder la manière dont les acteurs publics se saisissent de la notion de paysage, il faut rappeler que les stratégies des acteurs ne dépendent pas d'objectifs clairs et précis : elles se construisent en situation, elles sont liées aux atouts que les acteurs ont à leur disposition et aux relations dans lesquelles ils $s^{\prime}$ insèrent ${ }^{35}$. Or, «la littérature scientifique manque encore d'études de controverses déclenchées ou cristallisées autour de l'enjeu paysager; l'analyse fine des argumentaires et des jeux d'acteurs permettrait de mieux comprendre ce qui se joue dans ces situations, ce que l'on dit du paysage et ce que le paysage fait $\operatorname{dire}^{36}{ }$. En effet, les projets des acteurs, s'ils sont rarement clairs et cohérents, ne sont jamais absurdes. Il y a toujours un sens intrinsèque à l'élaboration d'un choix, qui répond à une stratégie

$\overline{35}$ Michel Crozier et Erhard Friedberg, L'acteur et le système, Paris, Seuil, coll. "Essais, Points», 1992.

36 Anne Sgard, "Une "éthique du paysage” est-elle souhaitable?», op. cit., p. 10. 
afin d'améliorer une capacité d'action et/ou de se préparer, se préserver, se garder des marges de manœuvre.

Ainsi, le paysage mis en politique relève d'abord du domaine des politiques publiques, largement regardées aujourd'hui par la recherche sur le paysage ${ }^{37}$. C'est en effet principalement à travers les instruments de l'action publique que sont observées les politiques de paysage aujourd'hui, plusieurs courants s'y croisant : l'évaluation de politiques paysagères (explicites ou implicites) ${ }^{38}$; l'implication des habitants dans la définition de politiques paysagères $^{39}$... Mais le paysage mis en politique est aussi celui de la pratique politique du paysage ${ }^{40}$. En regardant comment la notion de paysage est mobilisée localement, depuis l'émergence d'un problème à sa définition, à sa mise en pratique, à son évaluation, jusqu'à l'organisation du système d'acteurs et les discours véhiculés à cette occasion, c'est un autre versant de la recherche sur le paysage, à rapprocher des méthodes de la sociologie de l'action publique, qui s'ouvre.

Les travaux présentés ici résultent d'une méthode articulant la pratique du paysage par les acteurs publics locaux à la mobilisa-

37 Les thématiques des appels à projets de recherche émis par le ministère traduisent la façon dont le problème du paysage est perçu par l'État et les modèles de traitement du problème. Le premier programme concernait les «Politiques publiques et paysages, analyse, évaluation, comparaison» (1998), le second "Paysage et développement durable» (2005-2010), renouvelé une fois, proposait, dans sa deuxième édition, quatre axes de travail, parmi lesquels «Paysage et participation» et «Le Paysage, produit de l'économie - Services rendus par les paysages".

Nous renvoyons ici aux travaux de Didier Labat, notamment sa thèse, Le paysage, levier d'action dans la planification territoriale. Essai d'évaluation de la politique paysagère du SCOT de l'aire métropolitaine de Bordeaux, thèse de doctorat en sciences et architecture du paysage, AgroParis Tech/ENSP, Versailles, 2011. Les travaux de Patrick Moquay et al. sont aussi une référence importante : Patrick Moquay et al., «Une typologie des interventions intercommunales en matière de paysage», dans Martine Berlan-Darqué, Yves Luginbuhl, Daniel Terrasson (dir.), Paysages : de la connaissance à l'action, Versailles, Éditions Quae, coll. "Update Sciences and Technologies», 2007, p. 195-209.

Nous renvoyons ici à un travail récent : Alexis Pernet, Le grand paysage en projet entre trajectoires institutionnelles et territoires vécus..., op. cit.

40 Pierre Lascoumes et Patrick Le Galès, Sociologie de l'action publique, Paris, Armand Colin, 2009. 
tion du paysage dans les discours, qu'ils soient publics et officiels, ou individuels (voir les figures 3 et 4).

Les pratiques (voir la figure 3) se lisent à la fois dans l'espace concret (analyse paysagère) ainsi que dans la construction visible de l'espace politique (analyse politico-institutionnelle) et dans les documents publics accessibles : études, notes de cadrage, projets issus des services des collectivités territoriales.

Figure 3

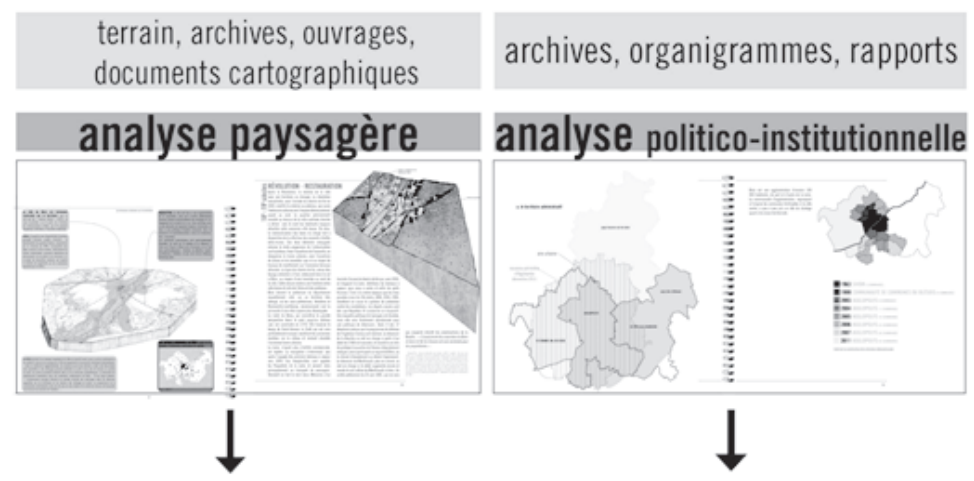

études, documents d'urbanisme, projets issus des services

\section{analyse des projets et des études}

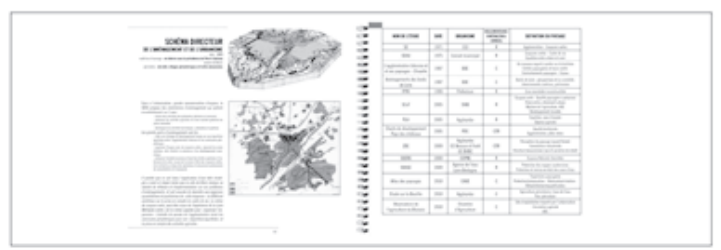

Schéma traduisant la méthode de collecte des pratiques des acteurs publics

Les discours (voir la figure 4) se lisent dans leur dimension publique, c'est-à-dire les communications officielles et les articles de presse locale, mais également dans la construction individuelle d'un discours sur le paysage. Il s'agit dans ce dernier cas de cerner comment les acteurs pensent leur rôle dans la mobilisation du paysage. Par l'intermédiaire d'entretiens semi-directifs avec les acteurs du territoire (élus, techniciens, représentants d'associations, 
représentants de l'État), on recherche également la construction du système sous-jacent, les représentations individuelles autant que les représentations collectives, portées et mises en politique.

Figure 4

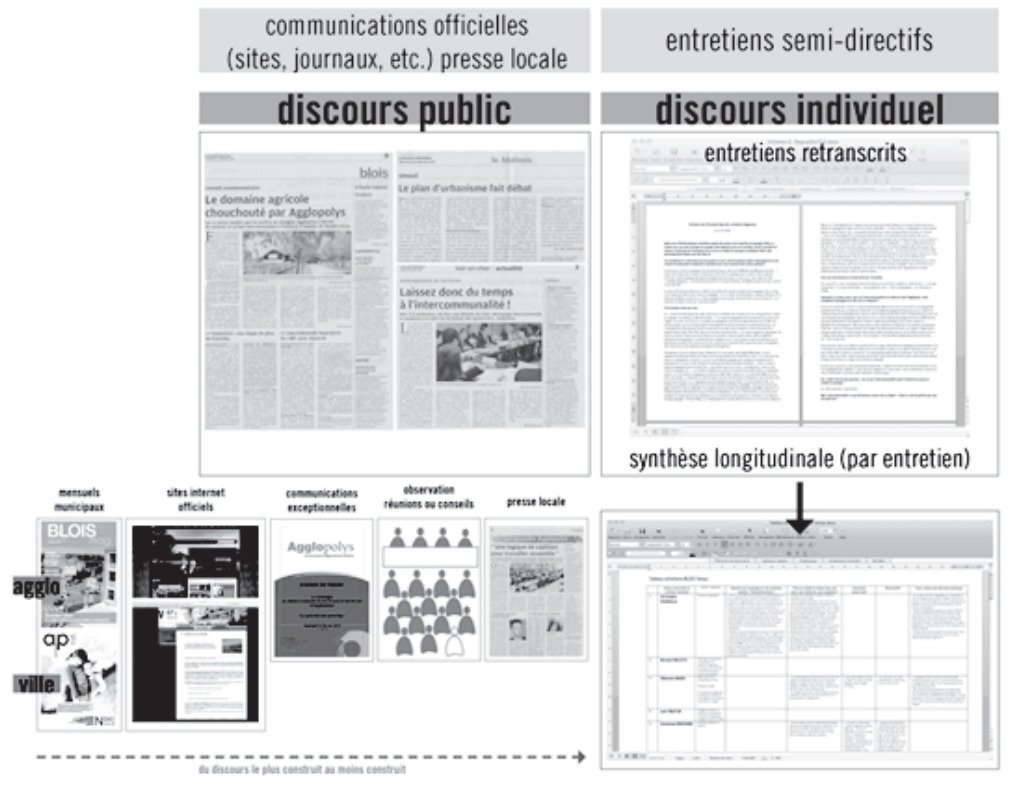

Schéma traduisant la méthode de collecte des discours des acteurs publics

Les exemples choisis présentent chacun une trajectoire singulière. C'est souvent dans la mise en place des premiers schémas directeurs dans les années 1970 que se trouvent les racines d'une trajectoire contemporaine, jusqu'aux discours récents suite aux élections municipales en 2008. Ces analyses font état d'une chronophotographie, jusqu'à un instant " $\mathrm{t}$ " (celui de la date de l'analyse), de la politique locale d'une ville moyenne sur des domaines assez divers, relatifs à la représentation du territoire et à son aménagement. 


\subsection{Des trajectoires singulières propres à chaque territoire}

Pour chaque ville moyenne, la mobilisation politique du paysage croise plusieurs domaines, que l'on peut tenter de distinguer en trajectoires, pour en comprendre le sens.

Tout d'abord, la trajectoire de la mobilisation du paysage semble dépendre de plusieurs facteurs locaux : l'histoire politique du territoire et l'organisation des acteurs dans un premier temps, notamment le poids des représentants de l'État et l'histoire de la construction intercommunale; d'un autre côté, les enjeux naturels du territoire, par exemple les risques majeurs (risque d'inondation principalement dans le cas des villes ligériennes); enfin, l'importance des politiques touristiques également, et de la tradition culturelle des édiles locaux, etc.

L'agglomération de Blois présente un intérêt particulier vis-àvis de la problématique, puisque la mobilisation du paysage y est assez importante actuellement. Elle peut ainsi être considérée comme un exemple-laboratoire. La comparaison avec Nevers et Saumur permet de mesurer ses originalités : en effet, à Nevers, le paysage est peu mobilisé, si ce n'est par les associations locales d'habitants en réaction à certaines tendances d'aménagement; à Saumur, au contraire, le paysage est fortement appelé, conjointement à la notion de patrimoine, et il demeure porté principalement par des acteurs supra-territoriaux, c'est-à-dire des représentants de l'État ou des représentants du Parc Naturel Régional Loire-Anjou-Touraine.

Après avoir analysé le détail des politiques urbaines et l'usage du paysage depuis les années $1970^{41}$, nous pouvons conclure à trois grands temps de l'histoire de la politique locale en termes de construction spatiale à Blois. La figure 5 présente le détail des politiques d'aménagement et les étapes de la construction politique intercommunale.

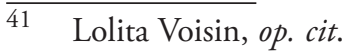


Figure 5

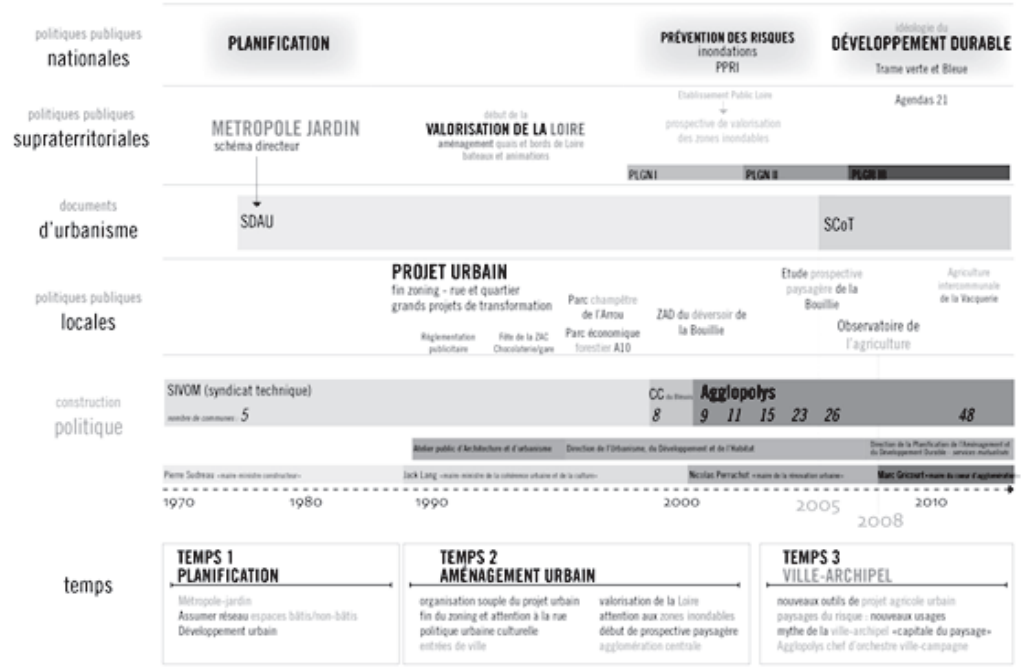

Frise chronologique détaillant les politiques locales ayant un lien avec la notion de paysage, sur l'agglomération de Blois

Le premier temps est celui de la planification, notamment du fait de l'influence des directives nationales à travers les documents d'urbanisme (Schéma Directeur d'Aménagement et d'Urbanisme) mais également dans la continuité de la tentative de métropole-jardin qui prévoyait d'organiser le développement de l'axe fluvial Orléans-Tours en un chapelet de villes entrecoupé de zones naturelles préservées de l'urbanisation ${ }^{42}$. C'est alors le tout début des syndicats mixtes à vocations techniques, rassemblant les communes proches du centre de l'agglomération. Au tout début des années 1990, la pratique des élus locaux intègre de nouveaux modèles. Tout d'abord, la valorisation de la Loire à l'échelle supra-territoriale renouvelle l'attention portée à l'espace urbain, d'abord par les bords de Loire. Mais c'est l'élection de Jack Lang et la mise en place de l'Atelier Public d'Architecture et d'Urbanisme, dirigé par Patrick Bouchain, qui soulignent

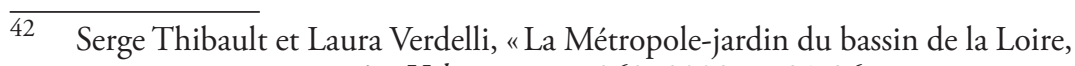
un projet anticipateur?» Urbanisme, $\mathrm{n}^{\circ} 363,2008$, p. 81-86. 
véritablement le début d'un nouveau temps, celui du "projet urbain». Outre "le retour à la rue et à l'échelle du quartier ${ }^{43}$ ", plusieurs projets lancés à cette époque transforment les frontières entre la ville et le hors-ville : le parc d'activité de la sortie de l'autoroute A10 par exemple, conçu comme un espace forestier évolutif selon les besoins économiques, dessine une entrée de ville originale et organisée (voir la figure 6). Puis, vers le début des années 2000, le durcissement des services de l'État vis-à-vis de la protection contre les risques d'inondation pose de nouvelles questions aux édiles des villes ligériennes, bâties pour la plupart de part et d'autre du lit majeur de la Loire, parfois même en son milieu (comme c'est le cas à Blois pour le quartier Vienne). À la fin des années 2000, c'est la problématique périurbaine qui entre pleinement dans les préoccupations locales. C'est le début d'un changement de discours et de pratiques sur les franges, en termes de valorisation de l'agriculture tout d'abord, d'habitat, de services, etc. Plusieurs outils d'aménagement sont créés, comme l'Observatoire du Blaisois qui recense les activités agricoles impactées par le fonctionnement urbain. L'échelle spatiale d'appréhension du territoire évolue considérablement : ce sont plus de quarante communes qui sont concernées par ces nouveaux instruments. Enfin, ce sont également les discours qui évoluent, avec le choix de nouveaux termes pour qualifier l'agglomération éclatée : les notions de ville-paysage, d'agglomérationarchipel $^{44}$ apparaissent après 2010 pour décrire l'ensemble composé de la ville-centre et de ses périphéries, étalées, diverses, comprenant des communes de plus en plus éloignées de Blois.

\footnotetext{
43 Selon les dires d'une urbaniste ayant vécu l'Atelier Public d'Architecture et d'Urbanisme.

44 Ces expressions font références aux modèles de développement créés par les grands paysagistes de la fin du XIX siècle, notamment Frederick Law Olmsted, architecte-paysagiste américain connu pour ses systèmes de parcs urbains (ou réseaux de parcs) au sein des métropoles nord-américaines, consacrés à l'expérience du paysage. Elles s'inspirent également de l'expérience de l'OREALM (Organisation d'Étude d'Aménagement de la Loire Moyenne) dans les années 1970, où le concept de métropole-jardin, reprenant l'alternance espaces bâtis / espaces naturels du corridor ligérien, était au cœur de la démarche de ce projet territorial entre Orléans et Tours. Ce projet reste en filigrane dans la mémoire des acteurs ligériens.
} 
À l'échelle de l'agglomération, certains élus locaux envisagent d'assumer pleinement la transformation de l'espace à laquelle ils assistent, impuissants, grâce à la lecture du paysage :

À la limite, dans la lecture strictement urbaine de la ville de Blois, la vision urbaniste peut suffire. Mais à partir du moment où l'une des grandes spécificités du territoire, c'est d'être à la fois dans l'urbain et le rural, $[. .$.$] d'avoir les zones d'articulation entre les zones denses et cette$ ville diffuse à traiter, on a besoin de la lecture du paysage ${ }^{45}$.

Figure 6

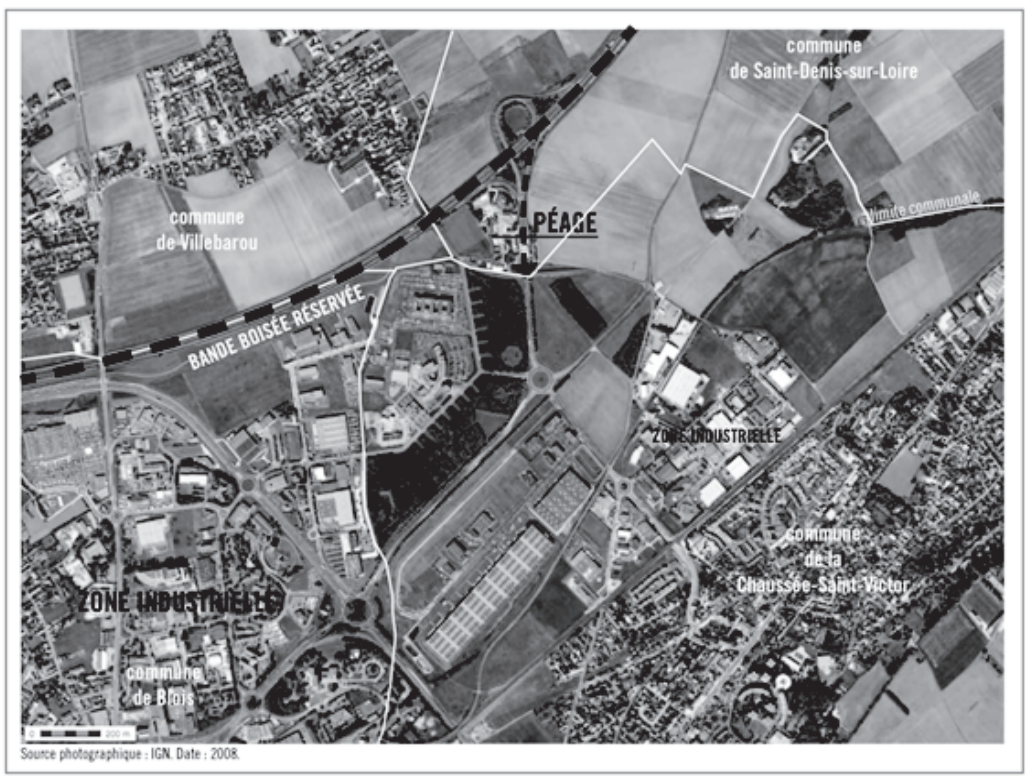

Situation du "parc A10", parc d'activités tertiaires situé près de la bretelle d'accès de l'autoroute A10, à l'est de Blois

Afin d'apporter des éléments de comparaison, la figure 7 montre l'évolution des politiques urbaines de l'agglomération de Nevers, pour laquelle la construction intercommunale n'arrive que tardivement, dans un contexte de résistance politique plus que de révélation d'un fonctionnement périurbain. À Nevers,

45 Extrait d'un entretien avec le président de la communauté d'agglomération, 2010. 
c'est davantage par la problématique fluviale (paysage d'exception $\mathrm{du}$ Bec d'Allier, risque d'inondation et valorisation de la zone inondable) que la dimension paysagère a été intégrée aux réflexions locales. Cependant, d'autres notions sont appelées, comme le développement durable (création en 2008 du Conseil Local de Développement Durable) et le patrimoine (Zone de Protection du Patrimoine Architectural, Urbain et Paysager de Nevers). Ainsi, le projet politique se concentre sur la ville-centre et le noyau urbain, alors que certains mouvements, portés par des habitants et des associations, amènent, depuis récemment, la question du paysage agricole sur la scène du débat public.

Figure 7

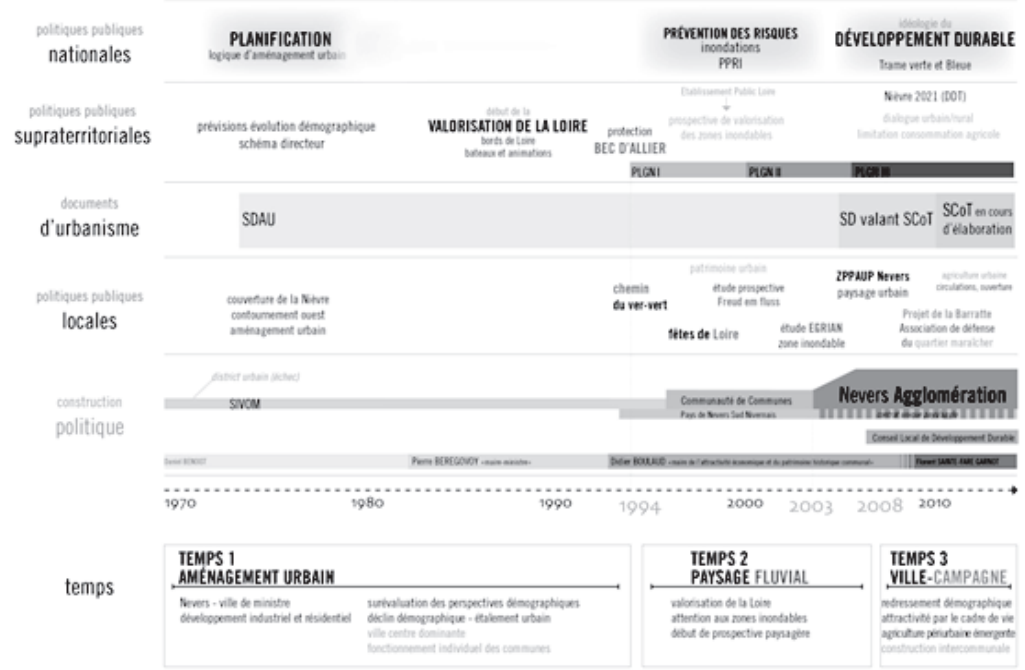

Frise chronologique détaillant les politiques locales ayant un lien avec la notion de paysage sur l'agglomération de Nevers

Ainsi, on peut s'interroger sur la manière dont le paysage participe pour certaines collectivités à un processus de territorialisation, actuellement en œuvre. Après avoir perdu les repères ancestraux divisant classiquement ville et campagne, établissant un pouvoir hiérarchique tenu par une mairie, les élus locaux sont conduits à chercher de nouveaux modèles. Peut-on voir dans la 
mobilisation du paysage un moyen de reconsidérer le territoire, de le redéfinir, de provoquer un processus de territorialisation plus que de fixer une nouvelle image, un nouveau périmètre?

Ainsi, la trajectoire locale de la mobilisation du paysage permet de cerner plusieurs facteurs par lesquels les acteurs publics engagent, d'une manière singulière, un processus de territorialisation $^{46}$. Tout d'abord, la relation à l'espace et la relation au temps sont deux facteurs évidents. Il est important d'y adjoindre un troisième facteur, qui serait la manière dont les acteurs s'organisent. Ce dernier est particulièrement intéressant pour mettre en évidence le processus de construction d'une territorialisation, et donc d'une entité territoriale construite par ses acteurs.

\section{Le paysage comme expérimentation locale pour gouverner la complexité territoriale?}

Maintenant, ouvrons de nouvelles perspectives pour l'action publique territorialisée. Vécu dans sa dimension politique, le paysage sert-il d'instrument pour comprendre, décrire, analyser et intervenir sur des phénomènes de plus en plus complexes? Le paysage est-il à l'échelle de l'agglomération blésoise comme une expérience pour gouverner certains aspects de la complexité territoriale? Nous avons mobilisé conjointement les trois facteurs de territorialisation, décrits plus haut, pour proposer des pistes de réflexion : tout d'abord, seront pointées quelques caractéristiques relatives à l'organisation entre acteurs et la mise en œuvre dans l'action d'une transversalité intersectorielle; ensuite nous développerons la capacité du paysage à mettre en récit le territoire de l'agglomération, notamment ses franges, en insistant sur les aspects temporels de l'action publique; enfin, nous regarderons plus précisément les politiques qu'on peut qualifier d'émergentes et qui tendent à produire une forme de cohérence d'ensemble pour l'action publique territoriale.

46 Pierre Dérioz, Philippe Béringuier et Anne Élisabeth Laques, «Mobiliser le paysage pour observer les territoires : quelles démarches, pour quelle participation des acteurs?", Développement durable et territoires, vol. 1, n ${ }^{\circ} 2$, septembre 2010, [en ligne] http://developpementdurable.revues.org/index8526. html, site consulté en mars 2011. 
2.1. La mise en ouvre d'une transversalité intersectorielle et d'une cohérence territoriale

Le propos n'est pas ici de donner au paysage la faculté de renouveler la démocratie, ce qui est pourtant avancé par ailleurs ${ }^{47}$. Il s'agit seulement d'observer que la mobilisation du paysage semble permettre la mise en œuvre d'une certaine transversalité dans l'action publique locale.

Tout d'abord, nous pouvons constater la mise en œuvre de nouvelles échelles de projet, plus complexes, par exemple autour de la question de l'agriculture périurbaine ou des zones inondables. La question de l'échelle d'intervention est constante chez les acteurs publics ${ }^{48}$. Ils sont constamment en train de jouer avec différents niveaux d'actions et différentes structures d'acteurs. Le Président de la communauté d'agglomération de Blois décrit cette complication :

On est constamment en train de passer d'échelles à d'autres, entre l'échelle pour laquelle on est élu, entre l'échelle de gestion, plus large, entre l'échelle de contractualisation, entre l'échelle qui peut être encore plus large, comme le syndicat du SCoT, et ainsi de suite ${ }^{49}$.

De plus en plus, en fonction des problèmes abordés et des clés de lecture employées, les acteurs travaillent sur des échelles aux contours adaptés, au sein desquelles se structure un jeu d'acteurs spécifique. Ainsi, la démarche de projet territorialisé semble permettre "un décloisonnement des savoirs sectoriel $s^{50}$ ", notamment par la valorisation des acteurs de terrain, par exemple des habitants, des élus locaux, ou encore des membres d'associations locales. Ce sont de nouvelles organisations d'acteurs, rassemblés autour d'une échelle problématique. Sont-elles susceptibles de

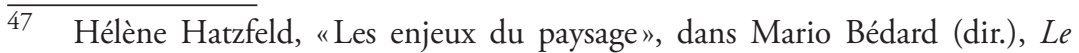
paysage, un projet politique, Québec, Presses de l'Université du Québec, 2009, p. 313-322.

48 Alexandre Moine, «Le territoire comme un système complexe : un concept opératoire pour l'aménagement et la géographie», Espace géographique, vol. 2006, no 2, 2006, p. 115-132.

49 Entretien avec le président d'Agglopolys le 17 décembre 2010.

50 Gilles Pinson, Projets et pouvoirs dans les villes européennes. Une comparaison de Marseille, Venise, Nantes et Turin, Thèse de doctorat en Science politique, Rennes, Université de Rennes 1, 2002. 
contrer une expertise délocalisée et banalisée? Cette démarche est souvent dénoncée par les acteurs locaux, qui sont obligés, dans le processus classique de projet, de faire appel à des bureaux d'études qui appliquent une méthode peu singularisée.

De plus, il semblerait que, par le paysage, le rapport aux experts est élargi. La comparaison du jeu d'acteurs autour de la question paysagère au sein des agglomérations (voir la figure 8) montre, dans le cas de Blois, un très fort recours à l'expertise extérieure à la communauté d'agglomération (à travers différents partenaires locaux comme l'École Nationale Supérieure de la Nature et du Paysage par exemple). C'est à travers une co-construction entre les professionnels "venus de l'extérieur» et les "experts locaux", de diverses formations que l'on multiplie les angles d'observation. L'interdisciplinarité d'une équipe permet l'hybridation des apports et leur partage. Les acteurs réunis autour d'une échelle problématique sont amenés à faire un travail de «traduction», nécessaire réinterprétation au regard des enjeux et des objectifs spécifiques aux différents acteurs locaux. Les techniciens des collectivités ont souvent des formations peu spécialisées (urbanistes, paysagistes, ingénierie territoriale, etc.), et deviennent alors des personnes ressources capables d'adaptation, essentielles au moment des premiers balbutiements de politiques transversales. Ces échanges et ces processus d'apprentissage se multiplient, à travers le nombre de scènes de forums et de débats, publics ou non. Ce sont, pour les mêmes acteurs, des scènes alternatives aux arènes que sont les scènes politisées, dans lesquelles est davantage sollicitée la connaissance fine du territoire ${ }^{51}$.

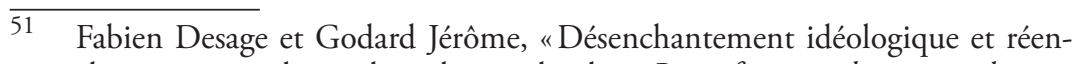
chantement mythique des politiques locales", Revue française de science politique, vol. 55, no 4, 2005, p. 633-661. 
Figure 8
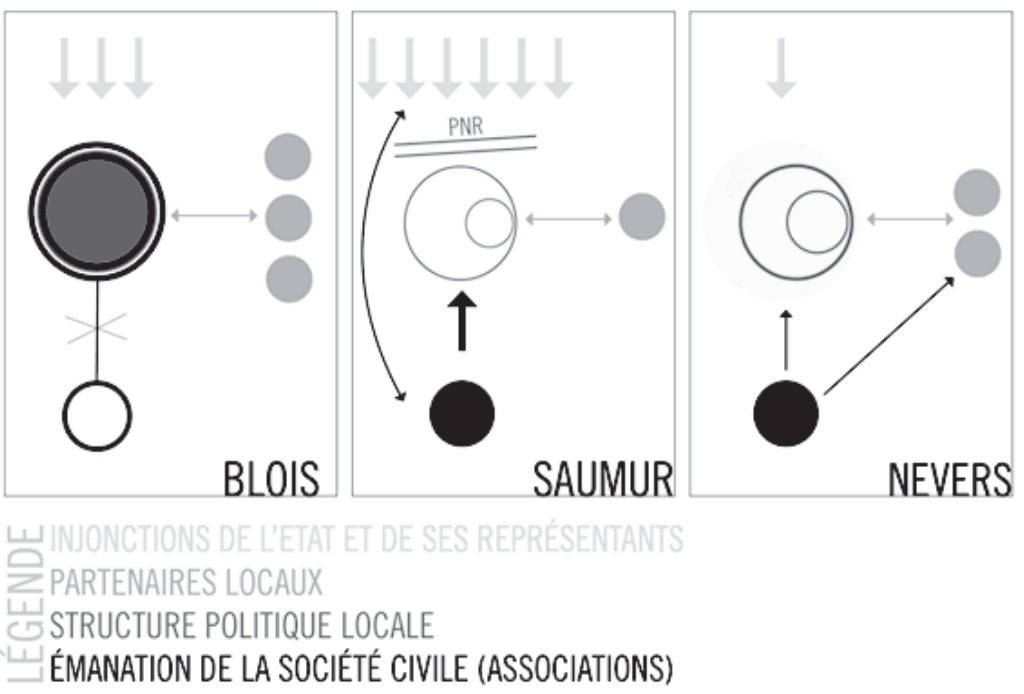

Représentation schématique des jeux d'acteurs comparés entre Blois, Nevers et Saumur. Le nombre d'éléments graphiques représente une grande implication des acteurs du "type " concerné (par exemple les partenaires ou les services de l'État). La structure politique locale apparaît dans les cercles à gauche au centre des trois tableaux; le nombre de cercles et les teintes correspondent aux échelons impliqués dans la mobilisation du paysage (ville, agglomération, syndicat du SCoT) et l'échelle de cette mobilisation.

Aussi, au sein des intercommunalités, les services restent souvent cloisonnés, par manque d'évolution depuis la création des EPCI, alors à vocation gestionnaire et technique (assainissement, déchets, transports collectifs, etc.). La prise en compte du paysage par les collectivités et sa traduction au sein de politiques territoriales bouleversent les sectorisations traditionnelles. "C'est difficile pour le politique de rassembler tous les responsables des différents secteurs pour voir comment on peut travailler ensemble, pour la valorisation d'un paysage, pour donner une identité ou donner une visibilité52.» Ainsi, à Nevers, cette situation est

52 Entretien avec une élue au Conseil général du Loir-et-Cher, le 13 septembre 2010. 
identifiée : «c'est encore très cloisonné, il n’y a aucune transversalité, mais cela change ${ }^{53} »$.

Certains techniciens notent un changement dans l'organisation du travail :

[Celle-ci] connaît une période de transition : avant, la hiérarchie du travail était verticale. Aujourd'hui, une nouvelle méthode horizontale se met en place. Celle-ci croise les services entre eux, de manière transversale. [...] La réforme générale des politiques de l'État a permis de fusionner des services qui ne travaillaient pas ensemble avant, comme le Conseil général. Cela est bénéfique mais intellectuellement difficile car rassembler des cultures différentes demande du temps ${ }^{54}$.

À Blois, la communauté d'agglomération a expérimenté cette transversalité depuis plusieurs années. L'un des élus témoigne :

Il y a une grande transversalité. La mobilité par exemple, cela implique le lancement d'un Plan de Déplacement Urbain sur l'ensemble de la communauté d'Agglo. [...] On a établi le comité de pilotage que je préside, qui réunit le vice-président Habitat et responsable du SCoT, le vice-président chargé du développement économique et le vice-président chargé du transport. Il y a donc une première transversalité. On y rajoute le maire de Blois et une série d'adjoints ou de conseillers municipaux de Blois qui s'occupent de voirie, transport, etc. J'ai voulu un maire d'une commune rurale du Sud de la Loire et une maire d'une commune rurale au nord de la Loire pour compléter $[\ldots]^{55}$.

Selon lui, c'est entre autres la prise en compte du paysage qui opère ce décloisonnement : "Tout est imbriqué, on ne peut pas penser un aspect sans penser à tous les autres. C'est un bel exercice».

Le président du CAUE ${ }^{56}$ du Loir-et-Cher, interrogé sur cette question, ne s'arrête pas à la transversalité des compétences internes :

53 Entretien avec un membre du Conseil Local de Développement Durable de Nevers, par ailleurs engagé dans la défense de l'environnement, le 4 mai 2011.

54 Entretien avec un chargé de mission au Pays Nevers Sud Nivernais, le 27 mai 2011.

55 Extrait d'un entretien avec le maire d'une commune de la première couronne de l'agglomération de Blois, et par ailleurs vice-président à la communauté d'agglomération de Blois, le 20 juillet 2010.

56 Conseil d'Architecture, d'Urbanisme et d'Environnement. 
Si le politique décide de faire une politique paysagère, d'aménagement ou ayant un impact sur le paysage, il pourrait tout de suite mettre deux "pieds» corollaires : tout d'abord, la formation des principaux acteurs de cette politique. Il ne s'agit pas de faire un acte de production linéaire très classique du projet. Il s'agit de former en amont les gestionnaires. Puis une action de communication auprès des habitants, pour qu'ils se l'approprient ${ }^{57}$.

À la communauté d'agglomération de Blois, le technicien chargé des risques, notamment celui d'inondation, confirme que "l'approche paysage est intéressante à ce stade de maturité de l'agglo", dans de nombreux domaines, "elle peut permettre de nous apprendre à croiser nos différents projets ${ }^{58}$ ". L'embauche récente d'un paysagiste au sein de la communauté d'agglomération de Blois, placé stratégiquement de manière transversale, immédiatement sous les ordres du Directeur général des services, corrobore cette volonté de ne placer le paysage dans aucun service spécifique, afin d'agir conjointement, dès l'amont, sur plusieurs domaines sectorisés.

Cette mutation en cours peut s'expliquer par les nouvelles questions posées aux collectivités locales, car :

toutes ces problématiques transversales qui apparaissent dans le débat local, comme la mobilité, le paysage, ou le développement durable, provoquent un nouveau type de gouvernance et des conflits dans la mise en commun des compétences. C'est un des problèmes émergents : mettre en commun des domaines culturellement différents. [...] La méthode consiste donc à organiser des organigrammes transversaux afin de lier l'ensemble des domaines, ce qui rejoint la philosophie d'Alain Berthoz. [...] L'enjeu est de gérer la complexité des milieux sans les sectionner ${ }^{59}$.

Il est intéressant de constater que les collectivités s'organisent au cas par cas, en fonction des projets et des stratégies locales. Les politiques environnementales, notamment les politiques de gestion de l'eau ou des espèces biologiques (bassins versants, Natura 2000), nécessairement fluides et transversales, ont souvent

\footnotetext{
$57 \quad$ Extrait d'un entretien avec le directeur du CAUE 41, le 2 juillet 2010.

58 Extrait d'un entretien avec le chargé de mission au Département Environnement Risques à la ville de Blois, le 29 juillet 2011.

59 Extrait d'un entretien avec un chargé de mission au PNSN, Nevers, le 27 mai 2011.
} 
été une étape primordiale de cette prise de conscience. De plus en plus, les idées d'économie et de cohérence influencent ces partages de compétences et ces relations entre services.

Par de nouveaux types de coopération, les services expérimentent des approches plus complémentaires, plus intégrées. Par exemple à Saumur, un projet sur les prairies inondables, initié par le service «Gestion et valorisation de l'espace» de la communauté d'agglomération, tente de croiser préoccupations esthétiques (patrimoine mondial de l'UNESCO), environnementales (maintien de prairies riches en biodiversité) et économiques (aide à l'économie de l'élevage). À Blois, cette capacité n'est pas sans rappeler le temps de l'atelier Public d'Architecture et d'Urbanisme (1989-1993), conçu sous la municipalité de Jack Lang comme une équipe autonome et transversale, mobile et flexible, apte à s'adapter à de nombreux types de problèmes et d'échelles d'intervention. La directrice de l'urbanisme des services mutualisés de la ville et de la communauté d'agglomération a débuté sa carrière dans cette équipe.

On a vécu de très bons moments quand on travaillait en régie, c'est très satisfaisant de faire, alors que, maintenant, on est dans le faire-faire. C'est un autre métier, une évolution. [...] L'atelier public des années 1990 a été un moment de foisonnement intense et extraordinaire, un bonheur absolu, un travail fou, une émulation collective ${ }^{60}$.

Quelques années auparavant, en 1994, cette urbaniste donnait ses impressions, du temps même du fonctionnement de l'Atelier public :

Ce qui est intéressant dans l'approche du projet urbain, c'est que c'est à la fois le très long terme avec de grands projets mais aussi la qualité du détail. C'est du quotidien, chaque jour. N'importe quel petit espace public a le droit d'être aussi bien traité qu'un grand. Tout compte, aussi bien une bordure de trottoir, la position d'une grille d'égout, toutes ces petites choses qui font, en fait, la beauté d'une ville ${ }^{61}$.

60 Extrait d'un entretien avec la directrice de l'urbanisme de Blois, le 15 septembre 2010.

61 Citée dans Régis Hermelin, Les politiques urbaines de Blois depuis 1989, Mémoire de maîtrise, Tours, Université François-Rabelais, 1995. 
Ainsi, ce sont également les allers et retours entre, d'une part, une attention aux petites choses et, d'autre part, la dimension stratégique sur les grands espaces, qui forgent la complémentarité d'une action publique territorialisée, et singularisée.

Enfin, le paysage semble permettre de dépasser une lecture uniquement technique du problème traité (par exemple la gestion du risque d'inondation et la sauvegarde des biens et des personnes) pour enclencher en même temps une réflexion sur l'espace et les usages. C'est une vision davantage politique qui émerge, qui sache à la fois intégrer les données techniques et scientifiques, recueillir les informations réelles nécessaires et élaborer un diagnostic pertinent, mais également ne pas s'éloigner de l'idée d'un changement, imaginer les usages et les économies qui sont susceptibles de s'agencer par exemple aux contraintes hydrauliques.

Il faut inventer une nouvelle instance démocratique, où l'élu, le citoyen prend en compte le savant. [...] C'est très stimulant de montrer comment aujourd'hui un certain nombre de problèmes environnementaux, comment la biodiversité, par exemple, ne peut pas être concernée par notre organisation politique, qui est à la fois limitée dans le temps, le cycle électoral, et également limitée dans l'espace, puisque les grands problèmes environnementaux n'ont aucun rapport avec notre problème de découpage politico-administratif ${ }^{62}$.

Ainsi, par une remise en question des pratiques sectorisées, c'est le potentiel de mise en cohérence d'une action publique locale qui est soulevée.

\subsection{La mise en récit du territoire par ses franges}

Finalement, s'opère un changement de regard sur l'espace du territoire, notamment par son appréhension "par les bords». En effet, la mobilisation du paysage sert de support à un nouveau récit entre acteurs publics, mais aussi vis-à-vis d'une communauté extérieure. Et ce sont principalement les franges, les espaces

62 Extrait d'un entretien avec le président de la Communauté d'agglomération de Blois, le 17 décembre 2010. 
incertains de la périurbanisation, les espaces de marge influencés par l'agglomération, qui soutiennent ce récit.

Par le biais de la notion de paysage, se joue un essai sémantique et planificateur pour s'intégrer à ce que serait une ville contemporaine assumée, c'est-à-dire une ville qui comprenne à la fois un centre urbain et ses faubourgs traditionnels, eux-mêmes au cœur du débat dans les années 1990, avec également une nouvelle périphérie, des espaces incertains, aux franges des agglomérations, pour lesquels on n'a encore ni modèle théorique ou scientifique, ni système politique établi, devenus pourtant le centre d'attention des élus locaux dans les années 2010.

Perpétuellement redéfinies par les trajectoires résidentielles et les mobilités quotidiennes des individus et par les tendances économiques, ces franges des villes moyennes, qui ne sont ni délimitées dans l'espace, ni précisées dans des représentations institutionnelles, se cherchent une définition : puisqu'elles ne sont ni ceci ni cela, ni ville ni campagne, ne sont-elles que les espaces de la dégradation et de la «France moche ${ }^{63}$ "? La menace que représente cette réalité, encore peu connue dans sa singularité, interroge profondément les modèles classiques : le devenir d'une agriculture devenue périurbaine, les capacités de mobilité, le développement d'un urbanisme détendu, l'espace social abandonné aux véhicules, sont autant de «nouveaux compromis urbains $^{64}$ ». Sont-ils de potentielles ressources pour l'action territoriale, grâce peut-être à leur souplesse, leur capacité de mutation, et aussi du fait de nouvelles esthétiques? En effet, le paysage n'est pas seulement dévolu à une dénonciation spatiale, souvent issue

63 Nous renvoyons ici au titre de couverture du périodique Télérama: Xavier de Jarcy et Vincent Remy, "Comment la France est devenue moche», Télérama, $\mathrm{n}^{\circ} 3135$, février 2010. L'anthropologue Éric Chauvier, habitant de ces périphéries décriées, avait répondu par un court manifeste Contre Télérama (Paris, Allia, 2011). Il y dénonce une violence de jugement, issue d'une centralité qui se voudrait souveraine et seule juge d'une esthétique collective. Un deuxième dossier spécial dans Télérama ( $\left.{ }^{\circ} 3268,2012\right)$, dénonce le cauchemar à venir de populations précaires, loin de tout et dépendantes des énergies.

64 François Ascher, Les nouveaux compromis urbains : lexique de la ville plurielle, La Tour-d'Aigues, Éditions de l'Aube, 2008. 
des habitants, d'association, d'intellectuels, d'artistes, et d'acteurs politiques. Il est également la raison d'une réalité sociale qui devient visible dans les bouleversements spatiaux qu'elle opère. Les acteurs de l'aménagement passent alors par le paysage pour décrire ces franges et porter un discours positif et encourageant ${ }^{65}$.

Outre cette relecture par les franges, le paysage est également mobilisé pour influencer la temporalité de l'action publique, et finalement la capacité projective des acteurs publics locaux. C'est alors le rapport au temps qui se reconfigure.

En effet, on observe par le paysage un attachement fort des acteurs locaux à l'héritage du passé. Le paysage physique est à la fois le témoin de mutations socio-économiques en cours, livre ouvert sur le présent. Il est également la résultante d'interactions passées entre une société et son milieu, inscrivant la société actuelle dans les traces de son passé. Le choix d'une référence passée n'est jamais anodin pour une personne publique. Le passé est en effet souvent mobilisé, à travers les politiques patrimoniales ${ }^{66}$, comme, dans une dimension moins nationale et plus diffuse, à travers les multiples politiques de développement territorial. Ainsi, le paysage s'avère être support de la réappropriation d'une histoire locale plus ou moins réinventée. Cette histoire revisitée servirait à légitimer l'action présente, à produire des signes d'ancrage. Les acteurs reconstruisent, par ce processus, un discours sur le passé, selon leur logique et leurs objectifs. Il ne s'agit donc pas d'un passé anecdotique, mais d'un passé choisi, par les acteurs, de manière individuelle et/ou collective.

Cet usage du passé s'accompagne d'une revalorisation moins des clichés que des pratiques, des économies et de l'ingéniosité des $"$ anciens ${ }^{67}$ ». Cette lecture produit comme une archéologie, sur des espaces qui ont été la plupart du temps fortement

65 Martin Vanier, «La périurbanisation comme projet», Métropolitiques, 2011, [en ligne] http://www.metropolitiques.eu/La-periurbanisation-comme-projet. html, site consulté en février 2012.

66 Pesant particulièrement lourd sur les collectivités appartenant au périmètre inscrit au patrimoine mondial de l'UNESCO, comme le sont Blois et Saumur.

67 François Hartog, Anciens, modernes, sauvages, Paris, Seuil, coll. «Points, Essais», 2008. 
remaniés pendant la seconde moitié du $\mathrm{XX}^{\mathrm{e}}$ siècle. À titre d'exemple, le maire d'une commune rurale, située au nord de l'agglomération blésoise en pleine Beauce, doit aujourd'hui faire face à des inondations pluviales. Les pratiques culturales ont changé, diminuant fortement les capacités d'infiltration des sols, et les structures de gestion de l'eau pluviale ont été arasées : "on n'a pas fait du tout attention à ce qu'on faisait, c'est valable [...] pour tout le territoire français, on n'a pas fait attention à l'agriculture, à l'urbanisation, à l'historique du ruissellement des eaux, aux pratiques culturales ${ }^{68}{ }$. Dans les études, le discours de la table rase provient davantage de l'expert, alors que l'on remarque que ce sont les acteurs locaux et les techniciens, en particulier ceux qui travaillent depuis longtemps sur le territoire, qui tiennent un discours davantage référencé au passé, sans pour autant donner de grandes orientations pour le long terme. Le paysage pourrait ainsi servir de lecture pour laquelle l'articulation entre la prise en compte d'une épaisseur, d'une lecture stratigraphique des lieux ${ }^{69}$, et une vision scénarisée de l'avenir envisagé. Cette épaisseur agit comme une ressource, une structure pour installer les bases d'une prospective pour le territoire.

Pour beaucoup d'acteurs, "le travail sur le paysage ou le jardin, c'est bien l'inscription dans la durée ${ }^{70} »$. Cette dimension s'est beaucoup diffusée par le biais de la crise écologique et la raréfaction des ressources, l'idée de durée se mêlant à celle de durabilité, dans une confusion des genres. Les acteurs locaux sont donc fortement encouragés, voire pressés, de "prévoir l'avenir", c'està-dire à la fois de prévoir les futures situations de crises, de les anticiper et d'intervenir pour préserver des ressources épuisables. Pour cela, de plus en plus d'études d'aménagement, qui suivaient traditionnellement le processus classique d'appel d'offres public, empruntent aujourd'hui un chemin plus long, plus sinueux, plus

\footnotetext{
68 Extrait d'un entretien avec le maire de Marolles, commune située à une dizaine de kilomètres au nord de Blois, sur le plateau de Beauce, le 5 janvier 2011. André Corboz et Sébastien Marot, Le Territoire comme palimpseste et autres essais, Besançon, L'imprimeur, 2001.

70 Extrait d'un entretien avec le président de la communauté d'agglomération de Blois, le 3 avril 2012.
} 
adaptatif. C'est le cas par exemple de l'étude sur le devenir du déversoir de la Bouillie à Blois, ou encore de l'étude EGRIAN sur les zones inondables à Nevers :

L'étude EGRIAN est très intéressante, mais on a commencé en 2007 et elle n'est toujours pas finie. Le gros problème pour ces études, ce sont les bureaux d'études car plus l'étude est longue et moins elle est rentable. Pour nous, collectivité, il ne faut pas griller les étapes. Il faut que ce soit une démarche engagée et qui continue. Alors, à la limite, pour nous, plus l'étude dure longtemps, plus cela nous permet de creuser plein de sujets, que l'on n'avait pas eu le temps de voir ${ }^{71}$.

Concernant le projet du déversoir de la Bouillie, dans le lit majeur de la Loire à Blois, c'est bien une projection dans le futur qui est envisagée, dans un processus itératif en fonction des réunions avec le comité de pilotage : «on imagine le territoire dans 20 ans, mais en restant ancré dans la réalité, non pas pour fournir une étude de style dont les retombées n'existeront jamais $^{72}{ }^{\prime}$. Le paysagiste qui a réalisé l'étude prospective sur le devenir du site de la Bouillie souligne que l'intérêt d'une telle démarche, c'est l'exploration, c'est "de rencontrer tout le monde». Un tel projet s'appuie à la fois sur l'existant et sur «le vécu des gens, des usagers, des gestionnaires ${ }^{73}$ " pour dessiner l'avenir de la zone inondable à long terme (voir la figure 9). Ici, la réflexion ne précède plus l'action, réflexion et action s'entremêlent davantage et se construisent au fur et à mesure, par exemple avec l'installation progressive de jardins ouvriers sur le périmètre, trois ans après le rendu de l'étude prospective. Ces phases d'interrogation sont nouvelles, parfois difficiles pour les élus, qui, contrairement aux techniciens, ne sont pas formés à la logique de projet : "ça ne reste quand même pas une habitude des collectivités d'être toujours dans ces phases de questionnement. On

71 Extrait d'un entretien avec le directeur de l'environnement et du développement durable à l'ADN, le 27 mai 2011.

72 Extrait d'un entretien avec le président du SIAB, le $1^{\text {er }}$ juin 2010.

73 Extrait d'un entretien avec l'ingénieur-paysagiste en charge de l'étude prospective du devenir du site de la Bouillie, le 6 juillet 2010. 
devrait le refaire, il est temps, mais je ne sens pas qu'ils soient prêts à se remettre dans cette logique ${ }^{74}$ ».

Figure 9

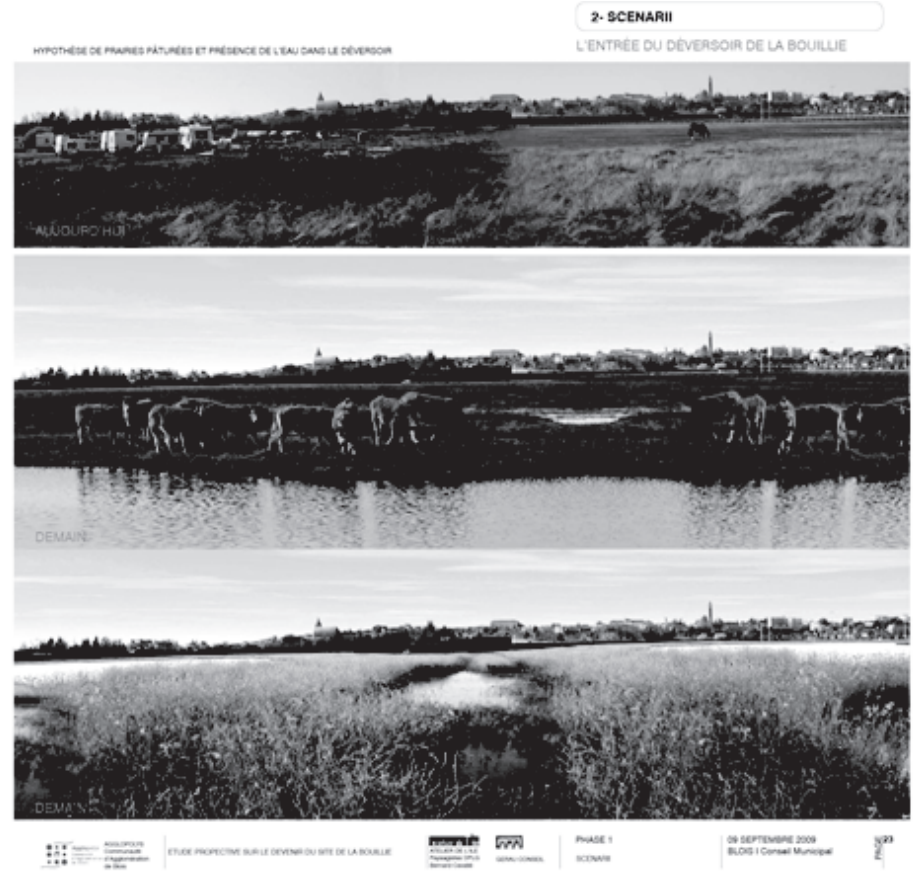

Étape préalable de l'étude prospective pour le devenir de la Bouillie. Le bureau d'étude présente au comité de pilotage des photomontages radicaux afin de faire émerger les représentations de l'avenir du déversoir et orienter les choix de scénarios. Source : Agglopolys; Réalisation : Atelier de l'île, 2009

L'approche par le paysage met en place les conditions de mise en œuvre d'une action globale. Il s'agit d'une forme de rupture méthodologique, où l'on apprivoise l'incertitude du futur en ouvrant au maximum des champs traditionnellement très étroits. D'ailleurs, le paysage, conçu comme processus, ne nie pas la mémoire des lieux et l'attachement à l'épaisseur, il permet de regarder le territoire comme un palimpseste ${ }^{75}$ des différents

74 Extrait d'un entretien avec un chargé de mission d'Agglopolys, le 6 septembre 2010.

75 À l'origine, le palimpseste est un parchemin dont la première écriture, grattée ou lavée, a fait place à un nouveau texte (Larousse, 2012). Il est utilisé par 
moments d'une société qui se sont succédés dans le temps et imbriqués dans l'espace. C'est une position encourageante pour les acteurs locaux, notamment les élus, "inscrits dans une culture politique et une pragmatique héritée», qui font confiance à l'existant, au "déjà-là», dans lesquels puiser leur discours public et leurs actions ${ }^{76}$. Cette ouverture vers l'avenir, en puisant dans les apprentissages du passé, permet de «légitimer les acteurs en les positionnant dans une filiation, de construire un collectif à travers le partage d'une histoire commune, d'ancrer une construction territoriale dans une histoire locale ${ }^{77}$ ». C'est par la mise en récit $^{78}$ de cette identité, par sa mise en intrigue, que l'action publique peut affirmer sa singularité et sa légitimité.

C'est la logique de l'action qui guide le récit, et non sa véracité historique. Car, à la différence de l'historien, l'acteur public local doit introduire sa propre logique d'action territoriale à ce récit. Ce dernier participe à rendre légitime l'action publique locale, puisque, par lui, elle seule semble connaître ce qui, du passé, a construit l'identité affichée du territoire, et que, par ce récit encore, l'action publique comprend qu'elle est capable d'anticiper et de projeter cette identité dans le futur. Cette mise en récit est en perpétuelle évolution, le récit se construit chemin faisant, en fonction des besoins de l'action publique; il peut se saisir de nouvelles figures, mettre en relief de nouvelles spécificités, définir de nouveaux "pas de temps» passés et futurs ou déformer les précédents, comme un moyen d'affirmer la construction collective d'un espace institutionnel en manque de reconnaissance.

Sébastien Marot à propos de la mémoire du territoire et de sa lecture par le paysage (L'art de la mémoire, le territoire et l'architecture, Paris, Villette, 2010). Anne Sgard, "Entre rétrospective et prospective. Comment reconstruire le récit du territoire?», 2008, [en ligne] http://www.espacestemps.net/en/ articles/entre-retrospective-et-prospective-en/, site consulté en mars 2011.

77 Ibid.

78 Cette notion de "mise en récit» a été développée dans des travaux récents, pour des sites viticoles inscrits à l'UNESCO : Serge Briffaud et Aline Brochot, Paysages d'exception, paysages au quotidien. Une analyse comparative de sites viticoles européens du Patrimoine mondial, Rapport de recherche remis au ministère de l'Écologie, de l'Énergie, du Développement durable et de la Mer, 2010 . 
Finalement, c'est par la marge que le paysage participe à la gouvernance de la complexité des territoires : par le "tiers-espace» qui se diffuse à plusieurs échelles réticulaires, pour lesquelles on accorde volontiers une absence de projet ou de vision politique; par le "tiers-temps» qui dépasse les logiques traditionnelles des temps de l'action publique (temps de l'étude, temps du mandat électoral, temps de l'administration, etc.). Ainsi, le paysage initie une reconnaissance politique de ce «tiers-espace / temps». Si ce n'est pas strictement à travers une nouvelle limite spatiale ou temporelle ou à travers la recherche d'une mise en périmètre, c'est plutôt par la mise en réseau, par l'articulation et la relation que l'on peut parler de nouveaux territoires d'action ${ }^{79}$. C'est par la définition lente d'un chant ${ }^{80}$, d'une épopée, d'un récit, dans la répétition d'éléments familiers, que les dimensions matérielles et immatérielles de l'espace sont apprivoisées, territorialisées à la manière d'une «ritournelle» publique ${ }^{81}$.

2.3. Des politiques émergentes à la repolitisation territoriale

Selon nos analyses, le paysage dans l'action publique locale se décline de plusieurs façons. Il y a les politiques spécifiquement paysagères, c'est-à-dire celles dont l'objectif principal est d'avoir une action directe sur le paysage ou de le valoriser, dans les discours par exemple, en tant qu'objet de représentation sociale et culturelle. Il y a les politiques thématiques faisant appel au paysage comme argument ou pour lesquelles le paysage est convoqué. Par exemple, une politique de prévention des risques d'inondation emprunte le chemin du paysage pour y appuyer la multiplicité des usages et le temps long de la prospective, comme c'est le cas pour le projet de la Bouillie. Mais il y a également les politiques où le paysage devient émergent. Ces dernières sont

\footnotetext{
79 Patrice Melé et Corinne Larrue, Territoires d'action. Aménagement, urbanisme, espace, Paris, L'Harmattan, coll. "Itinéraires géographiques", 2008. Par la multiplication de déclarations publiques par exemple ou par l'invention de nouveaux mots dans les discours publics.

81 Gilles Deleuze et Félix Guattari, Mille plateaux. Capitalisme et schizophrénie, Paris, Minuit, 1980.
} 
d'autant plus intéressantes qu'elles sont elles-mêmes des politiques émergentes.

Finalement, les politiques émergentes en question concernent, en filigrane, le statut des communautés d'agglomération. L'émergence de cette intercommunalité en tant que collectivité de projet, la place comme chef de file, comme moteur d'une stratégie spatiale, pour laquelle le site métropolitain n'est pas regardé comme un ensemble homogène mais plutôt comme un territoire de projet cohérent. Le territoire n'est pas compris comme un construit réel, actuel, mais davantage comme un potentiel (c'est-à-dire plein de potentialités). Par le paysage, les acteurs peuvent opérer un renversement conceptuel : c'est par les espaces ouverts, les espaces résiduels que la collectivité tente de reprendre le contrôle de l'agglomération au sens large. À partir de ce déplacement des espaces de pouvoir, c'est un «nouvel ordre territorial et urbain ${ }^{82}$ » que peuvent générer les acteurs publics.

Les communautés d'agglomération, d'abord construites sur des compétences techniques, gagnent progressivement des compétences et une dimension stratégique qui n'était pas au cœur de leur statut au départ. Les communes ont confié facilement les premières, pour des questions de coût de gestion et de mutualisation de moyens humains ${ }^{83}$. Aujourd'hui, les communautés d'agglomération sont l'échelon en gestation pour une nouvelle

\footnotetext{
82 Alberto Magnaghi, Le projet local, Bruxelles, Mardaga, coll. «Architecture + Recherches ", 2003.

83 Un directeur des services de la communauté d'agglomération de Nevers nous confiait cette évolution : "On a commencé les intercommunalités sur des compétences plutôt techniques, que les communes ont données facilement, parce que c'était compliqué, cher, etc. Aujourd'hui, on parle de conseillers communautaires qui changent dans la représentativité des élus. Par exemple, aujourd'hui, on a 80 élus, on va passer à $35[\ldots]$ avec une représentation qui n'est plus du tout celle qu'on avait puisqu'elle dépendra du poids démographique des communes. On a créé l'interco d'une manière participative, en disant aux petites communes qu'elles seront complètement représentées; aujourd'hui, elles n'auront plus rien à dire. La démocratie participative va en prendre un coup, mais, quelque part, c'est diplomatique et intelligent de la part de l'État... Les interco vont avoir de plus en plus de compétences et être de plus en plus influentes sur le territoire. Plus on a de compétences, plus on a de dotations. [...] On va prendre de nouvelles compétences, ici : la culture, le sport, peut-être l'urbanisme. Comme me disait une maire : "il me reste la
} 
gouvernance territoriale. Le paysage participe à la capacité "d'inventer" une imagerie territoriale, où l'agglomération devient la nouvelle référence pour parler d'entité urbaine, et donc pour imaginer sa gouvernance. Le discours sur le paysage, qui porte préférentiellement sur les franges, sur les grands réseaux structurels (réseau vert, réseau bleu, réseau d'espaces publics) qui se croisent et se superposent, permet à la communauté d'agglomération d'asseoir une légitimité politique, moins gestionnaire que gouvernant éclairé, voire utopiste. En mobilisant un discours à la fois subjectif et singularisant ${ }^{84}$, la collectivité devient non plus un outil d'application de politiques sectorielles, mais bien un acteur stratégique dans l'écheveau complexe des acteurs.

Il faut rappeler que les espaces ouverts sont le lieu de rencontre de plusieurs jeux de pouvoir, de plusieurs niveaux décisionnels. En effet, les politiques environnementales sont principalement le fait de mesures nationales, portées par l'État. Les collectivités ont peu de pouvoir dans ce domaine. En revanche, même si l'État est porteur de nombreuses mesures agri-environnementales, ce sont les acteurs publics locaux qui sont les moteurs d'un nouveau

salle des fêtes, les cérémonies et les cimetières". Mais c'est une évolution logique» (extrait d'un entretien du 27 mai 2011).

84 Guattari Félix et Suely Rolnik, Micropolitiques, Paris, Les empêcheurs de penser en rond, 2007 [1986]. Dans ce très riche et très morcelé ouvrage, Micropolitiques, le chapitre second, "Subjectivité et histoire", développe les notions de subjectivation et de singularisation. La singularisation consiste à être en capacité de se singulariser, par rapport à une uniformisation en cours des individus, de leurs désirs et de leurs manières d'être. La subjectivation concerne plus précisément la capacité pour un individu de construire sa propre lecture et son propre jugement de situations complexes. «Il faut que chacun s'affirme dans la position singulière qu'il occupe, qu'il la fasse vivre, qu'il l'articule avec d'autres processus de singularisation, et qu'il résiste à toutes les entreprises de nivellement de la subjectivité» (p. 71). La micropolitique de Félix Guattari se définit comme «une analytique des formations de désir dans le champ social». Cette attention à toute forme de contact, de relation à l'autre, est capable de provoquer un nouvel agencement, et de nouveaux processus de singularisation qui évitent le retour à la normativité. Ces micropolitiques sont la condition de l'émergence des potentiels, de leur apparition et de leur disparition. L'attention portée à l'émergence du discours des acteurs politiques, et à envisager qu'on puisse y trouver la naissance de nouvelles formes de singularisation, revient à faire un pas de côté par rapport aux discours à tendance dogmatique. 
regard sur l'agriculture périurbaine. L'intercommunalité dispose localement d'un rôle d'interface, entre des enjeux strictement urbains (souvent portés par la commune centre, généralement très urbaine) et des enjeux globaux, comme l'agriculture, les zones inondables ou encore la définition d'un territoire partagé. À travers son expertise propre et une certaine capacité de recul, les acteurs de la communauté d'agglomération, notamment les techniciens généralistes et les élus éclairés, deviennent capables de mettre à jour les nouveaux problèmes locaux, d'envisager des solutions innovantes et des partenariats inédits, bref, d'orchestrer une interface efficace dans les problématiques communautaires.

Figure 10

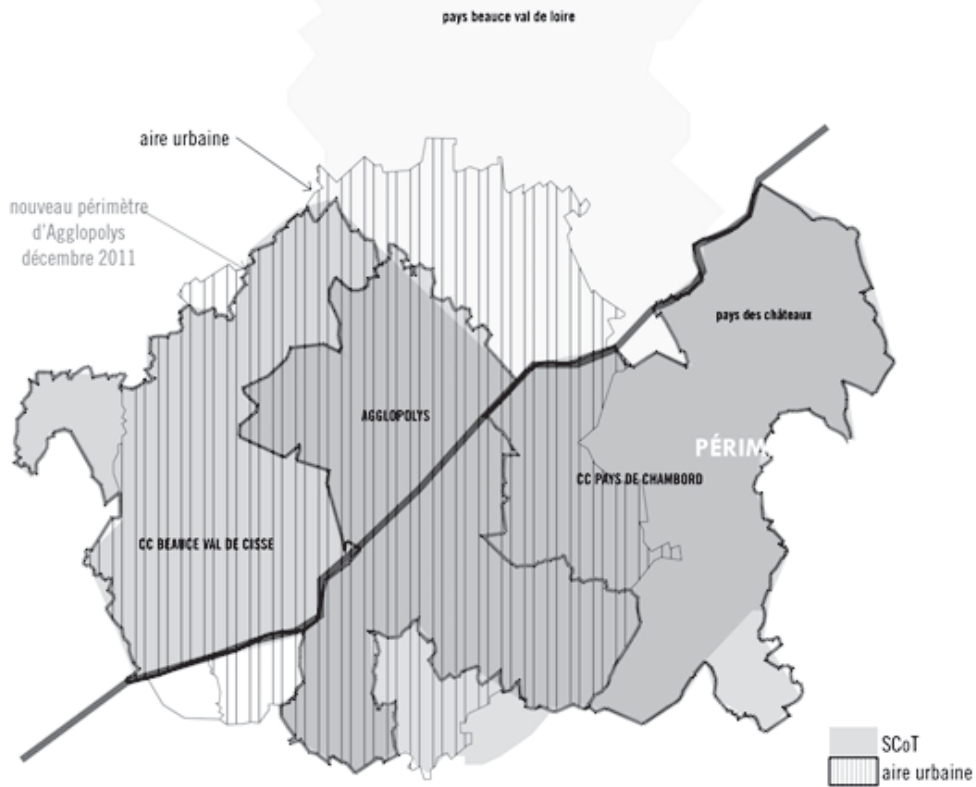

Carte de la construction de la communauté d'agglomération de Blois, semblable à l'aire urbaine définie par l'INSEE et proche du périmètre du SCoT

Ainsi, le paysage dirigerait le regard des acteurs communautaires soit vers les interstices, les délaissés des politiques publiques, soit vers des enjeux qui nécessitent une réflexion en réseau, 
comme la question de la gestion de l'eau, des populations animales ou humaines, du développement urbain intégré à la problématique agricole, etc. Le devoir des collectivités reviendrait alors à prendre en charge des affaires collectives, dans une interface entre urbain et rural, entre communes et échelons régionaux ou nationaux, entre amont et aval, et avec les collectivités voisines. Le paysage serait-il alors pour les collectivités un terrain d'expérimentation de la prise en charge de la complexité territoriale, sans dogmatisme et avec singularisation?

\section{Conclusion}

Grâce à la mobilisation du paysage, les acteurs locaux participent, toujours de manière singularisée, à la construction d'une transversalité et à la mise en cohérence des différents espaces d'action. Pour ce faire, il reste de nombreuses pistes pour amener les acteurs à mieux formuler et expliciter les objectifs poursuivis, et également à les communiquer. Cet apprentissage collectif doit faire l'objet d'approfondissements, ce que de futurs travaux de recherche pourront continuer de mettre à jour. La portée des recherches sur l'action publique territorialisée en train de se faire intéresse avant tout les acteurs locaux, dans un effort réflexif et prospectif.

Or, la question du paysage se situe au croisement d'une technicisation favorisée par l'État et de la définition d'un projet de territoire stratégique. Les services des collectivités regrettent souvent ces aspects technique et normatif qui concernent de nombreux domaines, jusquà celui des outils de planification. Lorsque le paysage est traité comme un domaine d'experts ou de spécialistes, il fait l'objet d'une approche parfois dogmatique et, de ce fait, souvent peu réappropriée par les acteurs locaux. "Surtout si l'expert, payé cher et venu de loin, vient nous dire ce que nous savons déjà! ${ }^{85}$ " Malgré de nombreux efforts pour partager des études paysagères, comme les atlas de paysage, celles-ci restent peu utilisées, souvent davantage perçues comme

85 D'après l'entretien avec le vice-président en charge de la planification et de l'aménagement des territoires à l'agglomération de Saumur, le 27 août 2012. 
des cautions vis-à-vis d'acteurs supra-territoriaux (services de l'État principalement) et comme des mises en conformité réglementaires que comme de véritables outils stratégiques de conception partagée. Or, «on ne peut pas laisser le paysage comme une affaire de spécialistes ${ }^{86} »$.

Finalement, nous proposons de considérer le paysage avant tout comme un processus, plus qu'un état figé. Le paysageprocessus encourage à "faire des économies», à inventer de nouvelles stratégies adaptées à la situation locale, à déceler, à mobiliser et à réemployer des forces déjà existantes. Selon Jacques Rancière, la politique est une forme d'expérience, qui "porte sur ce qu'on voit et ce qu'on peut en dire, sur qui a la compétence pour voir et la qualité pour dire, sur les propriétés des espaces et les possibles du temps ${ }^{87}$ ». Les acteurs publics peuvent parler du paysage comme d'un espace de l'action publique rendue visible, où le collectif partage rencontres et controverses. Chaque acteur peut savoir que le paysage reste pourtant le fruit d'une relation singulière, individuelle, personnelle, le fruit de sa propre «ritournelle». Cette posture est au cœur de ce qui constitue le rôle d'un acteur politique ${ }^{88}$, notamment dans son appréhension de l'espace public. Selon l'architecte Patrick Bouchain, l'espace est public lorsqu'il réunit les conditions et les médiations constitutives d'une existence politique ${ }^{89}$. L'espace public est un espace politique car, lorsque l'espace est privé de tout lieu commun, il meurt. C'est par le réseau des espaces publics que la démocratie dispose de l'espace nécessaire à son expression, espace néanmoins mis à mal par un aménagement du territoire trop mécanique, où le singulier est renié. La planification quantitative et modélisée enferme et coupe les espaces et les populations; la normalisation sépare davantage qu'elle ne rassemble. Par le pouvoir de singularisation de l'approche paysagère, l'acteur

\footnotetext{
$86 \quad$ Entretien avec le directeur du CAUE 41, le 2 juillet 2010.

87 Jacques Rancière, Le Partage du sensible. Esthétique et politique, Paris, La Fabrique, 2000.

88 Hannah Arendt, Qu'est-ce que la politique?, Paris, Seuil, 1959.

89 Patrick Bouchain, Construire autrement, Arles, Actes Sud, 2006.
} 
public est en capacité d'infléchir ${ }^{90}$ la règle et d'employer l'espace comme un allié pour inventer à nouveau, là où elles ont disparu, les conditions du politique. C'est d'ailleurs en assumant cette dimension politique du paysage que pourra être évitée la confiscation de la notion par certains aux dépens d'autres, que pourront être repoussés les risques, sur le plan éthique, que comporte intrinsèquement cette notion, ainsi que ceux d'un retour facile à la norme.

Cette singularisation par la notion de paysage semble permettre de nouvelles formes de territorialités pour les acteurs publics : par un engagement vis-à-vis des objets qui composent le territoire, des relations qui les gouvernent, des incertitudes de l'action et des imprévisibles; elle est en même temps une revalorisation des pratiques de gouvernement local qu'opèrent ses préposés. Ce pourrait bien être un levier pour l'apprivoisement de la complexité spatiale et sociale des territoires contemporains, l'acceptation des échelles imbriquées nécessaires à une action territoriale cohérente et innovante, mais aussi pour une relecture éminemment politique de la notion de territoire. L'implication relative des communautés d'agglomération dans la prise en compte et le gouvernement de la complexité des territoires agit comme un signal de cette perspective pour les territoires et comme des expérimentations par le bas de reterritorialisation politique.

90 Mais aussi de recréer une norme, c'est un autre danger. 


\section{Bibliographie}

Arendt, Hannah, Qu'est-ce que la politique?, Paris, Seuil, 1959.

Ascher, François, Les nouveaux compromis urbains: lexique de la ville plurielle, La Tour-d'Aigues, Éditions de l'Aube, 2008.

Barraqué, Bernard, Paysage et administration, Mission de la recherche urbaine, MEDD-Direction de la Nature et des Paysages, 2005 [1985].

Bédard, Mario, «Habiter la Terre. Le paysage, un projet politique», dans Le paysage : un projet politique, p. 1-12. Québec, Presses universitaires du Québec, 2009.

Behar, Daniel, Martin Vanier et Philippe Estèbe, "Pôles métropolitains : du "faire territoire" au "faire politique", où la nouvelle bataille de l'interterritorialité», Métropolitiques, 18 mai 2011, [en ligne] http:// www.metropolitiques.eu/Poles-metropolitains-du-faire.html, site consulté en septembre 2012.

Berque, Augustin, «Douter du paysage», dans Jean-Pierre Le Dantec (dir.), Jardins et Paysage, Textes critiques de l'Antiquité à nos jours, Paris, Larousse, 1996.

Besse, Jean-Marc, Le goût du monde. Exercices de paysage, Arles, Actes Sud/ ENSP, collection «Paysage», 2009.

Blanc, Nathalie et Sylvie Glatron, «Du paysage urbain dans les politiques nationales d'urbanisme et d'environnement", L'Espace géographique, vol. 2005, no 1, tome 34, p. 65-80.

Bouchain, Patrick, Construire autrement, Arles, Éditions Actes Sud, 2006.

Briffaud, Serge et Aline Brochot, Paysages d'exception, paysages au quotidien. Une analyse comparative de sites viticoles européens du Patrimoine mondial, Rapport de recherche remis au ministère de l'Écologie, de l'Énergie, du Développement durable et de la Mer, 2010.

Caille-Cattin, Catherine, Le paysage, de l'information à l'élaboration d'une culture paysagère. Vers des médiations paysagères au service des acteurs de l'aménagement des territoires, Thèse de doctorat en géographie Université de Franche-Comté, 2005.

Éric Charmes, «Les politiques paysagères comme supports éthiques de la gentrification", Actes du colloque De la connaissance des paysages à l'action paysagère, 2 au 4 décembre 2004, Bordeaux, Cemagref, 2004.

Charmes, Éric, La ville émiettée, essai sur la clubbisation de la vie urbaine, coll. «La ville en débat», Paris, Presses universitaires de France, 2011.

Chauvier, Éric, Contre Télérama, Paris, Allia, 2011.

Corboz, André et Sébastien Marot, Le Territoire comme palimpseste et autres essais, Besançon, L'imprimeur, 2001. 
Crozier, Michel et Erhard Friedberg, L'acteur et le système, Paris, Seuil, coll. «Essais, Points », 1992.

Davodeau, Hervé, "Les atlas de paysages français ou les difficultés de concilier l'approche sensible et l'approche scientifique», Rivista Geografica Italiana, vol. 2009, n 2, 2009, p. 173-194, 2009.

Deleuze, Gilles et Félix Guattari, Mille plateaux. Capitalisme et schizophrénie, Paris, Minuit, 1980.

Dérioz, Pierre, Philippe Béringuier et Anne, Élisabeth Laques, «Mobiliser le paysage pour observer les territoires : quelles démarches, pour quelle participation des acteurs?", Développement durable et territoires, vol. 1, $\mathrm{n}^{\circ} 2$, 2010, [en ligne] http://developpementdurable.revues.org/ index8526.html, site consulté en mars 2011.

Desage, Fabien et Godard Jérôme, "Désenchantement idéologique et réenchantement mythique des politiques locales", Revue française de science politique, vol. 55, $\mathrm{n}^{\circ}$ 4, 2005, p. 633-661.

Folléa, Bertrand, Guide des plans de paysage, des chartes et des contrats, Ministère de l'Aménagement du territoire et de l'environnement, 2001.

Fortin, Marie-Josée, «Le paysage, cadre d'évaluation d'une société réflexive», dans Martine Berlan-Darque, Yves Luginbühl, Daniel Terrasson (dir.), De la connaissance des paysages à l'action paysagère, Versailles, Éditions Quae, 2007, p. 223-231.

Guattari Félix et Suely Rolnik, Micropolitiques, Paris, Les empêcheurs de penser en rond, 2007 [1986].

Hartog, François, Anciens, modernes, sauvages, Paris, Seuil, coll. «Points, Essais», 2008.

Hatzfeld, Hélène, «Les enjeux du paysage», dans Mario Bédard (dir.), Le paysage, un projet politique, Québec, Presses de l'Université du Québec, 2009, p. 313-322.

Hermelin, Régis, Les politiques urbaines de Blois depuis 1989, Mémoire de maîtrise, Tours, Université François-Rabelais, 1995.

Jarcy, Xavier de et Vincent Remy, "Comment la France est devenue moche", Télérama, n 3135, février 2010.

Labat, Didier, "La mise en ouvre des politiques paysagères: quand la décision publique est confrontée aux échelles de définition. L'analyse des Proximités comme outil de compréhension de la coordination de l'action paysagère», Projets de paysage, 2011, [en ligne] http://www. projetsdepaysage.fr/fr/_la_mise_en_oeuvre_des_politiques_paysageres_ quand_la_decision_publique_est_confrontee_aux_echelles_de_definition, site consulté en janvier 2012. 
Labat, Didier, Le paysage, levier d'action dans la planification territoriale. Essai d'évaluation de la politique paysagère du SCOT de l'aire métropolitaine de Bordeaux, thèse de doctorat en sciences et architecture du paysage, AgroParis Tech/ENSP, Versailles, 2011.

Lascoumes, Pierre et Patrick Le Galès, Sociologie de l'action publique, Paris, Armand Colin, 2009.

Lajarge, Robert, "Pas de territorialisation sans action (et vice-versa)", dans Martin Vanier (dir.), Territoire, territorialité, territorialisation... et après?, Rennes, Presses universitaires de Rennes, 2009, p. 193-204.

Latour, Bruno, Nous n'avons jamais été modernes. Essai d'anthropologie symétrique, Paris, La Découverte, 1991.

Magnaghi, Alberto, Le projet local, Bruxelles, Mardaga, coll. «Architecture + Recherches", 2003.

Marcel, Odile, "L'espace citoyen. Le paysage comme outil de l'action démocratique», dans Mario Bédard (dir.), Le paysage: un projet politique, Québec, Presses de l'Université de Québec, 2009, p. 225-251.

Marot, Sébastien, L'art de la mémoire, le territoire et l'architecture, Paris, Villette, 2010.

Melé, Patrice et Corinne Larrue, Territoires d'action. Aménagement, urbanisme, espace, Paris, L'Harmattan, coll. "Itinéraires géographiques", 2008.

Moine, Alexandre, «Le territoire comme un système complexe: un concept opératoire pour l'aménagement et la géographie ", Espace géographique, vol. 2006, n 2, 2006, p. 115-132.

Patrick Moquay et al., "Une typologie des interventions intercommunales en matière de paysage», dans Martine Berlan-Darqué, Yves Luginbuhl, Daniel Terrasson (dir.), Paysages : de la connaissance à l'action, Versailles, Éditions Quae, coll. "Update Sciences and Technologies», 2007, p. 195-209.

Nadou, Fabien, Intermédiation territoriale et spatialisation des activités économiques. Cohérences et contradictions de l'action publique locale. Investigation par la planification stratégique, thèse de doctorat, Tours, Université François-Rabelais, 2013.

Pecqueur, Bernard, "Qualité et développement territorial : l'hypothèse du panier de biens et de services territorialisés ", Économie rurale, $\mathrm{n}^{\circ} 261$, 2001, p. 37-49.

Pernet, Alexis, Le grand paysage en projet entre trajectoires institutionnelles et territoires vécus - L'expérience de l'atelier des paysages en vallée de l'Ance (Parc naturel régional Livradois-Forez, Auvergne, France), thèse de doctorat en géographie, Paris, Université Panthéon Sorbonne-Paris 1, 2011. 
Pinson, Gilles, Projets et pouvoirs dans les villes européennes. Une comparaison de Marseille, Venise, Nantes et Turin, Thèse de doctorat en Science politique, Rennes, Université de Rennes 1, 2002.

Rancière, Jacques, Le Partage du sensible. Esthétique et politique, Paris, La Fabrique, 2000.

Sgard, Anne, "Une "éthique du paysage" est-elle souhaitable?» Vertigo, vol. 10, $\mathrm{n}^{\circ}$ 1, Dossier Ethique et Environnement à l'aube du XXIe siècle: la crise écologique implique-t-elle une nouvelle éthique environnementale?, 2010, [en ligne] http://vertigo.revues.org/9472, site consulté en mars 2011.

Sgard, Anne, «Entre rétrospective et prospective. Comment reconstruire le récit du territoire?», Espacestemps.net, 2008, [en ligne] http://www. espacestemps.net/en/articles/entre-retrospective-et-prospective-en/, site consulté en mars 2011.

Télérama, $\mathrm{n}^{\circ} 3268,2012$.

Thibault Serge et Laura Verdelli, «La Métropole-jardin du bassin de la Loire, un projet anticipateur?», Urbanisme, no 363, 2008, p. 81-86.

Vanier, Martin, "Qu'est-ce que le tiers espace? Territorialités complexes et construction politique», Revue de Géographie Alpine, tome 88, nº 1 , 2000, p. 105-113.

Vanier, Martin, "La périurbanisation comme projet», Métropolitiques, 2011, [en ligne] http://www.metropolitiques.eu/La-periurbanisationcomme-projet.html, site consulté en février 2012.

Voisin, Lolita, La mobilisation du paysage par les acteurs publics locaux, un enjeu stratégique de territorialisation? Réflexions en Loire Moyenne : Blois, Nevers, Saumur, Thèse de doctorat en aménagement de l'espace-urbanisme, Tours, Université François-Rabelais, 2013.

Walter, François, Les figures paysagères de la nation. Territoire et paysage en Europe (XVI $-X X^{e}$ siècles), Paris, Éditions de l'EHESS, 2004. 